IMA Journal of Numerical Analysis (2014) 34, 1328-1360

doi:10.1093/imanum/drt041

Advance Access publication on November 22, 2013

\title{
Moment equations for the mixed formulation of the Hodge Laplacian with stochastic loading term
}

\author{
FRANCESCA BONIZZONI* \\ MOX - Dipartimento di Matematica "F. Brioschi”, Politecnico di Milano, via Bonardi 9, 20133 \\ Milano, Italy and CSQI - MATHICSE, École Polytechnique Fédérale de Lausanne, Station 8, CH-1015 \\ Lausanne, Switzerland \\ *Corresponding author: francesca.bonizzoni@polimi.it \\ ANNALISA BUFFA \\ Istituto di Matematica Applicata e Tecnologie Informatiche del CNR, via Ferrata 1, 27100 Pavia, Italy \\ annalisa@imati.cnr.it \\ AND \\ FABIO NOBILE \\ MOX - Dipartimento di Matematica "F. Brioschi”, Politecnico di Milano, via Bonardi 9, 20133 \\ Milano, Italy and CSQI - MATHICSE, École Polytechnique Fédérale de Lausanne, Station 8, CH-1015 \\ Lausanne, Switzerland \\ fabio.nobile@epfl.ch
}

[Received on 12 November 2012; revised on 15 July 2013]

\begin{abstract}
We study the mixed formulation of the stochastic Hodge-Laplace problem defined on an $n$-dimensional domain $D(n \geqslant 1)$, with random forcing term. In particular, we focus on the magnetostatic problem and on the Darcy problem in the three-dimensional case. We derive and analyse the moment equations, that is, the deterministic equations solved by the $m$ th moment $(m \geqslant 1)$ of the unique stochastic solution of the stochastic problem. We find stable tensor product finite element discretizations, both full and sparse, and provide optimal order-of-convergence estimates. In particular, we prove the inf-sup condition for sparse tensor product finite element spaces.
\end{abstract}

Keywords: finite element exterior calculus; Hodge Laplacian; mixed finite elements; uncertainty quantification; stochastic partial differential equations; moment equations; sparse tensor product approximation.

\section{Introduction}

Many engineering applications are affected by uncertainty. This uncertainty may be due to incomplete knowledge of the input data or some intrinsic variability of them. For example, if we model single or multi-phase flow in a porous medium, randomness arises in the permeability tensor, due to the impossibility of a full characterization of conductivity properties of subsurface media, but also in the source term, typically pressure gradients or impervious boundaries. See for example Tartakovsky \& Neuman (1998), Guadagnini \& Neuman (1999a,b), Zhang (2002), Riva et al. (2006), Babuška et al. (2007) and Franssen et al. (2009). Similar situations appear in many other applications, such as combustion flows, earthquake engineering, biomedical engineering and finance. Probability theory provides an effective tool to include uncertainty in the model: the uncertain parameters are modelled as random variables or random fields with known probability laws. 
In this work, we focus on the linear Hodge-Laplace problem in a mixed formulation with stochastic forcing term described as an $L^{m}$-integrable process and homogeneous boundary conditions. This problem includes the magnetostatic and electrostatic equations as well as the Darcy problem for monophase flows in saturated media. The exterior calculus is a theoretical approach that, using tools from differential geometry, allows one to simultaneously treat many different problems. In particular, the Hodge Laplacian $\mathrm{d} \delta+\delta \mathrm{d}$, where $\delta$ is the formal adjoint of the exterior derivative $\mathrm{d}$, maps differential $k$-forms to differential $k$-forms and unifies some important second-order differential operators, such as the Laplacian and curl-curl problems arising in electromagnetics. For more details, see Arnold et al. $(2006,2010)$ and Christiansen et al. (2011).

The solution of the mixed formulation of the stochastic Hodge-Laplace problem is a couple $(u, p)$ of random fields taking values in a suitable space of differential forms. The description of these random fields requires knowledge of their moments. A possible approach is to compute the moments by the Monte Carlo method in which, after sampling the probability space, the deterministic partial differential equation (PDE) is solved for each sample and the results are combined to obtain statistical information about the random field. This is a widely used technique, but it features a very slow convergence rate.

In recent years some improvements have been proposed. We mention the multilevel Monte Carlo method (see e.g. Heinrich, 2001; Giles, 2008a,b; Barth et al., 2011; Cliffe et al., 2011; Teckentrup et al., 2013) for applications to stochastic PDEs) and the quasi Monte Carlo method (see e.g. Niederreiter, 1992; Caflisch, 1998; Graham et al., 2011; Kuo et al., 2012 for applications to stochastic PDEs).

An alternative strategy is to directly calculate the moments of interest of the stochastic solution without doing any sampling. Indeed, the aim of the present work is to derive the moment equations, that is, the deterministic equations solved by the $m$-points correlation functions of the stochastic solution, show their well-posedness and propose a stable sparse finite element approximation.

The stochastic problem has the form

$$
T\left[\begin{array}{l}
u \\
p
\end{array}\right]=\left[\begin{array}{l}
f_{1} \\
f_{2}
\end{array}\right] \quad \text { a.e. in } D
$$

where $T$ is a deterministic second-order linear differential operator (the Hodge-Laplace operator), $D$ is a domain in $\mathbb{R}^{n}$ and the forcing terms $f_{1}(\omega, x), f_{2}(\omega, x)$ are random fields, with $x \in D, \omega \in \Omega$ and $\Omega$ indicating the set of possible outcomes. The $m$ th moment equation involves the tensor product operator $T^{\otimes m}:=\underbrace{T \otimes \cdots \otimes T}_{m \text { times }}$ and the forcing term is given by the $m$-points correlation function of the couple $\left[\begin{array}{l}f_{1} \\ f_{2}\end{array}\right]$.

We start by proving the well-posedness of the $m$ th moment equation. Although this comes easily from a tensorial argument, we also present a direct proof of the inf-sup condition for the tensor operator $T^{\otimes m}$. This proof will be a key tool to show the stability of a sparse finite element approximation.

Concerning the numerical approximation of the $m$ th moment equation, a tensorized finite element (FE) approach for the numerical approximation of the moment equations is viable only for small $m$, as the number of degrees of freedom increases exponentially in $m$. For large $m$ one should consider instead sparse approximations (see e.g. Schwab \& Todor, 2003; Bungartz \& Griebel, 2004; Schwab \& Gittelson, 2011 and the references therein). We consider both full tensor product (FTP) and sparse tensor product finite element (STP-FE) approximations, and prove their stability using the tools from the finite element exterior calculus. In particular, the stability of an FTP approximation is a simple consequence of a tensor product argument. On the contrary, a tensor product argument does not apply if sparse tensor 
product approximations are considered and a direct proof of the inf-sup condition is needed, and will be proved in Section 6. We also provide optimal order-of-convergence estimates both for the full and the sparse approximations.

The originality of this work consists in the characterization of the inf-sup operator $P$ for the deterministic Hodge-Laplace operator $T$ such that $P^{\otimes m}$ is an inf-sup operator for the tensorized operator $T^{\otimes m}$. Using this result, we are able to prove the stability of sparse approximations of tensorized mixed problems, using advanced techniques such as a tensorial version of the GAP property (see Buffa, 2005). Only after finishing and submitting the work did we became aware of the work by Hiptmair et al. (2012), which treats the Maxwell cavity source problem using similar techniques.

The analysis on well-posedness and stable discretization for the $m$-points correlation problem developed in this work will be necessary to analyse more complex situations with randomness appearing in the operator itself instead of simply in the right-hand side. This case can be treated for small randomness by a perturbation approach (Taylor or Neumann expansions, see e.g. Tartakovsky \& Neuman, 1998; Guadagnini \& Neuman, 1999a,b; Riva et al., 2006 from the hydrology literature, and Babuška \& Chatzipantelidis, 2002; Cohen et al., 2011; Bonizzoni \& Nobile, 2013; Bonizzoni, 2013) and is currently under investigation. The outline of the paper is the following: in Section 2, we recall the Sobolev spaces of differential forms and the main results on the mixed formulation of the Hodge-Laplace problem in the deterministic setting, stating the well-posedness of the problem and translating it into the language of PDEs using proxy fields. In Section 3, we consider the stochastic counterpart of the mixed Hodge-Laplace problem and we prove the well-posedness of its weak formulation. Section 4 is dedicated to the analysis of the moment equations where we provide, in particular, the constructive proof of the inf-sup condition for the tensor product operator $T^{\otimes m}$. In Section 5, we focus on two problems of particular interest from the point of view of applications: the stochastic magnetostatic equations and the stochastic Darcy problem. In Section 6 we provide both full and sparse finite element discretizations for the deterministic $m$ th moment problem; we prove their stability and optimal order-of-convergence estimates. Conclusions are given in Section 7.

\section{Sobolev spaces of differential forms and the deterministic Hodge-Laplace problem}

In this section, we first recall the main concepts and definitions concerning finite element exterior calculus and Sobolev spaces of differential forms, which generalize the classical Sobolev spaces, inspired by Arnold et al. (2006, Section 2). We prove the inf-sup condition for the mixed formulation of the Hodge-Laplace problem, providing a choice of test functions different from the classical one proposed in Arnold et al. (2006). This will be needed later on to prove the equivalent inf-sup condition for the $m$-points correlation problem. Finally, in the three dimensional case, we interpret the Hodge-Laplace problem in term of proxy fields, and we translate it into the language of PDEs, with the aim of showing that this general setting includes some important problems of practical interest.

\subsection{Sobolev spaces of differential forms}

The natural setting is a sufficiently smooth finite-dimensional manifold $D$ with or without boundary. For our purposes, we can restrict ourselves to the particular case of an $n$-dimensional bounded domain $D \subset \mathbb{R}^{n}$ with boundary denoted by $\partial D \subset \mathbb{R}^{n-1}$. In this way, at each point $x \in D$ the tangent space is naturally identified with $\mathbb{R}^{n}$ and we make this assumption throughout the paper. We denote by $\operatorname{Alt}^{k} \mathbb{R}^{n}$, $1 \leqslant k \leqslant n$ the space of alternating $k$-linear maps on $\mathbb{R}^{n}$. Clearly, $\mathrm{Alt}^{0} \mathbb{R}^{n}=\mathbb{R}$ and $\mathrm{Alt}^{n} \mathbb{R}^{n}=\mathbb{R}$, and the unique element in $\mathrm{Alt}^{n} \mathbb{R}^{n}$ is a volume form $\operatorname{vol}_{n}$. We recall the wedge product $\wedge: \mathrm{Alt}^{k} \mathbb{R}^{n} \times \mathrm{Alt}^{l} \mathbb{R}^{n} \rightarrow$ 
$\mathrm{Alt}^{k+l} \mathbb{R}^{n}$ and the inner product $(\cdot, \cdot)_{\mathrm{Alt}^{k} \mathbb{R}^{n}}: \mathrm{Alt}^{k} \mathbb{R}^{n} \times \mathrm{Alt}^{k} \mathbb{R}^{n} \rightarrow \mathbb{R}$ for $k+l \leqslant n$. Starting from this inner product, the Hodge star operator $\star: \mathrm{Alt}^{k} \mathbb{R}^{n} \rightarrow \mathrm{Alt}^{n-k} \mathbb{R}^{n}$ is defined: $u \wedge \star w=(u, w)_{\mathrm{Alt}^{k} \mathbb{R}^{n}} \operatorname{vol}_{n}$.

A differential $k$-form on $D$ is a map $u$ which associates to each $x \in D$ an element $u_{x} \in \mathrm{Alt}^{k} \mathbb{R}^{n}$. We denote by $\Lambda^{k}(D)$ the space of all smooth differential $k$-forms on $D$. The wedge product of alternating $k$-forms may be applied pointwise to define the wedge product of differential forms: $(u \wedge w)_{x}=u_{x} \wedge w_{x}$. The exterior derivative $\mathrm{d}^{k}$ maps $\Lambda^{k}(D)$ into $\Lambda^{k+1}(D)$ for each $k \geqslant 0$ and it is defined as

$$
\mathrm{d}^{k} u_{x}\left(v_{1}, \ldots, v_{k+1}\right)=\sum_{j=1}^{k+1}(-1)^{j+1} \partial_{v_{j}} u_{x}\left(v_{1}, \ldots, \hat{v}_{j}, \ldots, v_{k+1}\right), \quad u \in \Lambda^{k}(D)
$$

$v_{1}, \ldots, v_{k+1} \in \mathbb{R}^{n}$, where the hat is used to indicate a suppressed argument. The exterior derivative satisfies the key property $\mathrm{d}^{k+1} \circ \mathrm{d}^{k}=0$ for all $k$. The coderivative operator $\delta^{k}: \Lambda^{k}(D) \rightarrow \Lambda^{k-1}(D)$ is the formal adjoint of the exterior derivative and it is defined by

$$
\star \delta^{k} u=(-1)^{k} \mathrm{~d}^{n-k} \star u, \quad u \in \Lambda^{k}(D) .
$$

To lighten the notation, in the following we omit the superscript $k$ and denote $\mathrm{d}^{k}$ and $\delta^{k}$ simply by $\mathrm{d}$ and $\delta$, respectively, when no ambiguity arises. The trace operator $\operatorname{Tr}: \Lambda^{k}(D) \rightarrow \Lambda^{k}(\partial D)$ is defined as the pullback of the inclusion $\partial D \hookrightarrow D$. We denote by vol the unique volume form in $\Lambda^{n}(D)$ such that, at each $x \in D, \operatorname{vol}_{n}$ is the unique form associated with $\operatorname{Alt}^{n} \mathbb{R}^{n}$. Given two differential $k$-forms on $D$, it is possible to define their $L^{2}$ inner product as the integral of their pointwise inner product in $\mathrm{Alt}^{k} \mathbb{R}^{n}$ :

$$
(u, w):=\int_{\mathrm{D}}\left(u_{x}, w_{x}\right)_{\mathrm{Alt}^{k} \mathbb{R}^{n}} \mathrm{vol}=\int_{\mathrm{D}} u \wedge \star w, \quad u, w \in \Lambda^{k}(D)
$$

In the following, we will denote by $\|\cdot\|$ the norm induced by the $L^{2}$ inner product $(\cdot, \cdot)$. The following integration by parts formula holds:

$$
(\mathrm{d} u, v)=(u, \delta v)+\int_{\partial D} \operatorname{Tr}(u) \wedge \operatorname{Tr}(\star v), \quad u \in \Lambda^{k}(D), v \in \Lambda^{k+1}(D) .
$$

The completion of $\Lambda^{k}(D)$ in the norm induced by the scalar product (2.2) defines the Hilbert space $L^{2} \Lambda^{k}(D)$. The Sobolev space of square-integrable $k$-forms whose exterior derivative is also square integrable is given by

$$
H \Lambda^{k}(D)=\left\{u \in L^{2} \Lambda^{k}(D) \mid \mathrm{d} u \in L^{2} \Lambda^{k+1}(D)\right\} .
$$

It is a Hilbert space equipped with the inner product

$$
(u, w)_{H \Lambda^{k}}:=(u, w)+(\mathrm{d} u, \mathrm{~d} w) .
$$

In analogy with $H \Lambda^{k}(D)$, it is possible to define the Hilbert space

$$
H^{*} \Lambda^{k}(D):=\left\{u \in L^{2} \Lambda^{k}(D) \mid \delta u \in L^{2} \Lambda^{k-1}(D)\right\} .
$$


Let $\partial D=\bar{\Gamma}_{\mathrm{D}} \cup \bar{\Gamma}_{\mathrm{N}}, \Gamma_{\mathrm{D}} \cap \Gamma_{\mathrm{N}}=\emptyset$. As is standard (Arnold et al., 2006), the spaces (2.4) and (2.5) can be endowed with boundary conditions:

$$
\begin{aligned}
& H_{\Gamma_{\mathrm{D}}} \Lambda^{k}(D):=\left\{u \in H \Lambda^{k}(D)|\operatorname{Tr}(u)|_{\Gamma_{\mathrm{D}}}=0\right\}, \\
& H_{\Gamma_{\mathrm{N}}}^{*} \Lambda^{k}(D):=\left\{u \in H^{*} \Lambda^{k}(D)|\operatorname{Tr}(\star u)|_{\Gamma_{\mathrm{N}}}=0\right\} .
\end{aligned}
$$

With the spaces defined in (2.6) and the exterior derivative operator, we can construct the $L^{2}$ de Rham complex:

$$
0 \rightarrow H_{\Gamma_{\mathrm{D}}} \Lambda^{0}(D) \stackrel{\mathrm{d}}{\rightarrow} \cdots \stackrel{\mathrm{d}}{\rightarrow} H_{\Gamma_{\mathrm{D}}} \Lambda^{n}(D) \rightarrow 0
$$

Since $\mathrm{d} \circ \mathrm{d}=0$, we have

$$
\mathfrak{B}_{k} \subseteq \mathfrak{Z}_{k},
$$

where $\mathfrak{B}_{k}$ is the image of $\mathrm{d}$ in $H_{\Gamma_{\mathrm{D}}} \Lambda^{k}(D)$ while $\mathfrak{Z}_{k}$ is the kernel of $\mathrm{d}$ in $H_{\Gamma_{\mathrm{D}}} \Lambda^{k}(D)$.

The following orthogonal decomposition of $L^{2} \Lambda^{k}(D)$, known as Hodge decomposition, holds:

$$
L^{2} \Lambda^{k}(D)=\mathfrak{B}_{k} \oplus \mathfrak{B}_{k}^{\perp}
$$

where $\mathfrak{B}_{k}^{\perp}$ is the $L^{2}$ complement of $\mathfrak{B}_{k}$.

We define two projection operators $\pi^{\perp}$ and $\pi^{\circ}$ as follows:

$$
\begin{aligned}
& \pi^{\perp}: \mathfrak{B}_{k} \oplus \mathfrak{B}_{k}^{\perp} \rightarrow \mathfrak{B}_{k}^{\perp}, \quad v=\mathrm{d} v^{\circ}+v^{\perp} \mapsto v^{\perp}, \\
& \pi^{\circ}: \mathfrak{B}_{k} \oplus \mathfrak{B}_{k}^{\perp} \rightarrow \mathfrak{B}_{k-1}^{\perp}, \quad v=\mathrm{d} v^{\circ}+v^{\perp} \mapsto v^{\circ} .
\end{aligned}
$$

Hence, given $v \in L^{2} \Lambda^{k}(D)$, it can be uniquely expressed as $v=\mathrm{d} \pi^{\circ} v+\pi^{\perp} v$. We recall a classical result in the theory of Sobolev spaces.

Lemma 2.1 (Poincaré inequality) There exists a positive constant $C_{\mathrm{P}}$ that depends only on the domain $D$ such that

$$
\|v\| \leqslant C_{\mathrm{P}}\|\mathrm{d} v\| \quad \forall v \in \mathfrak{Z}_{k}^{\perp}
$$

where $\mathfrak{Z}_{k}^{\perp}$ is the orthogonal complement of $\mathfrak{Z}_{k}$ in $H_{\Gamma_{\mathrm{D}}} \Lambda^{k}(D)$.

For the sake of simplicity, we consider only the case of geometries which are trivial from the topological point of view. More precisely, from now on, we make the following assumption.

Assumption 2.2 The domain $D \subset \mathbb{R}^{n}$ is bounded, Liptschitz and contractible. Its boundary $\partial D$ is given by the disjoint union of two open sets $\Gamma_{\mathrm{D}}$ and $\Gamma_{\mathrm{N}}$, with $\Gamma_{\mathrm{D}}, \Gamma_{\mathrm{N}} \neq \emptyset, \Gamma_{\mathrm{D}}$ contractible as well and with boundary sufficiently regular (at least piecewise $C^{1}$ ).

Under Assumption 2.2, $\mathfrak{B}_{k}^{\perp}=\mathfrak{B}_{k}^{*}$, where $\mathfrak{B}_{k}^{*}$ is the image of $\delta$ in $H_{\Gamma_{\mathrm{N}}}^{*} \Lambda^{k}(D)$. This relation is proved in the three-dimensional case in Fernandes \& Gilardi (1997) and generalizes to the $n$-dimensional case (see e.g. Massey, 1991).

From now on we make the following regularity assumption on the domain $D$, which will be needed to prove the stability of the numerical schemes we propose in this paper. 
Assumption 2.3 For every $0 \leqslant k \leqslant n$, there exists $0<s \leqslant 1$ such that

$$
H_{\Gamma_{\mathrm{D}}} \Lambda^{k}(D) \cap H_{\Gamma_{\mathrm{N}}}^{*} \Lambda^{k}(D) \subseteq H^{s} \Lambda^{k}(D)
$$

where $H^{s} \Lambda^{k}(D)$ is the space of differential $k$-forms with square-integrable partial derivatives of order at most $s$.

Inclusion (2.13) is verified for an s-regular domain such that $\Gamma_{\mathrm{D}}=\partial D$ and $\Gamma_{\mathrm{N}}=\emptyset$. In particular, if $\partial D$ is smooth, then $D$ is 1-regular, and if $\partial D$ is Lipschitz, then $D$ is $\frac{1}{2}$-regular. See Arnold et al. (2006) and the references therein. We assume the second inclusion to be verified in our more general setting where $\Gamma_{\mathrm{N}} \neq \emptyset$ and $\Gamma_{\mathrm{D}} \subsetneq \partial D$.

REMARK 2.4 The case of nontrivial topology can likely be treated following Arnold et al. (2010), but it would make the exposition of our results much more difficult.

Remark 2.5 We assume $\Gamma_{\mathrm{D}}, \Gamma_{\mathrm{N}} \neq \emptyset$, but the two limit cases treated in Arnold et al. (2006) can be considered with suitable modifications of our argument.

We end the section by introducing the following notation for two Hilbert spaces we will use later on:

$$
W_{k}:=\left[\begin{array}{c}
L^{2} \Lambda^{k}(D) \\
L^{2} \Lambda^{k-1}(D)
\end{array}\right], \quad V_{k}:=\left[\begin{array}{c}
H_{\Gamma_{\mathrm{D}}} \Lambda^{k}(D) \\
H_{\Gamma_{\mathrm{D}}} \Lambda^{k-1}(D)
\end{array}\right],
$$

endowed with the inner products $(\cdot, \cdot)_{W_{k}},(\cdot, \cdot)_{V_{k}}$ and the norms $\|\cdot\|_{W_{k}},\|\cdot\|_{V_{k}}$, respectively.

\subsection{Mixed formulation of the Hodge-Laplace problem}

The Hodge Laplacian is the differential operator $\delta \mathrm{d}+\mathrm{d} \delta$ mapping $k$-forms into $k$-forms and the HodgeLaplace problem is the boundary value problem for the Hodge Laplacian. We consider the mixed formulation of the Hodge-Laplace problem with variable coefficients, described in Arnold et al. $(2006,2010)$ and Christiansen et al. (2011), which allows one to include the Darcy problem (see Section 2.2.1). Given a non-negative coefficient $\alpha \in \mathbb{R}_{+}$and source terms $\left[\begin{array}{l}f_{1} \\ f_{2}\end{array}\right] \in W_{k}$, find $\left[\begin{array}{l}u \\ p\end{array}\right]$ such that

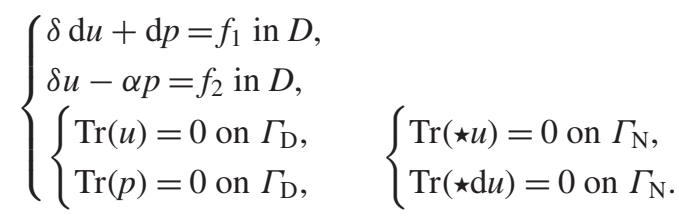

We introduce $T: V_{k} \rightarrow V_{k}^{\prime}$, the linear operator of order 2 represented by the matrix

$$
T:=\left[\begin{array}{cc}
\delta \mathrm{d} & \mathrm{d} \\
\delta & -\alpha \mathrm{Id}
\end{array}\right]=\left[\begin{array}{cc}
A & B^{*} \\
B & -\alpha \mathrm{Id}
\end{array}\right],
$$

where

$$
V_{k}^{\prime}=\left[\begin{array}{c}
\left(H_{\Gamma_{\mathrm{D}}} \Lambda^{k}(D)\right)^{\prime} \\
\left(H_{\Gamma_{\mathrm{D}}} \Lambda^{k-1}(D)\right)^{\prime}
\end{array}\right]
$$


is the dual space of $V_{k}$ defined in (2.14), the operators $A$ and $B$ are defined as

$$
\begin{aligned}
& A: H_{\Gamma_{\mathrm{D}}} \Lambda^{k}(D) \rightarrow\left(H_{\Gamma_{\mathrm{D}}} \Lambda^{k}(D)\right)^{\prime}, \quad\langle A v, w\rangle:=(\mathrm{d} v, \mathrm{~d} w), \\
& B: H_{\Gamma_{\mathrm{D}}} \Lambda^{k}(D) \rightarrow\left(H_{\Gamma_{\mathrm{D}}} \Lambda^{k-1}(D)\right)^{\prime}, \quad\langle B v, q\rangle:=(v, \mathrm{~d} q)
\end{aligned}
$$

and $B^{*}$ is the adjoint of $B$. Moreover, we introduce the linear operators $F_{1} \in\left(H_{\Gamma_{\mathrm{D}}} \Lambda^{k}(D)\right)^{\prime}$ and $F_{2} \in$ $\left(H_{\Gamma_{\mathrm{D}}} \Lambda^{k-1}(D)\right)^{\prime}$ defined as

$$
\begin{gathered}
F_{1}: H_{\Gamma_{\mathrm{D}}} \Lambda^{k}(D) \rightarrow \mathbb{R}, \quad F_{1}(v):=\left(f_{1}, v\right), \\
F_{2}: H_{\Gamma_{\mathrm{D}}} \Lambda^{k-1}(D) \rightarrow \mathbb{R}, \quad F_{2}(q):=\left(f_{2}, q\right) .
\end{gathered}
$$

The mixed formulation of the deterministic Hodge Laplacian with homogeneous essential boundary conditions on $\Gamma_{\mathrm{D}}$ and homogeneous natural boundary conditions on $\Gamma_{\mathrm{N}}$ is as follows.

\section{Deterministic Problem}

$$
\begin{gathered}
\text { Given }\left[\begin{array}{l}
F_{1} \\
F_{2}
\end{array}\right] \in V_{k}^{\prime}, \quad \text { find }\left[\begin{array}{l}
u \\
p
\end{array}\right] \in V_{k} \text { such that } \\
T\left[\begin{array}{l}
u \\
p
\end{array}\right]=\left[\begin{array}{l}
F_{1} \\
F_{2}
\end{array}\right] \text { in } V_{k}^{\prime} .
\end{gathered}
$$

THEOREM 2.6 For every $\alpha>0$, problem (2.21) is well posed, so that there exists a unique solution that depends continuously on the data. In particular, for every $\left[\begin{array}{l}u \\ p\end{array}\right] \in V_{k}$, take $\left[\begin{array}{c}v \\ q\end{array}\right]=P\left[\begin{array}{l}u \\ p\end{array}\right] \in V_{k}$, with

$$
P=\left[\begin{array}{cc}
\pi^{\perp} & \mathrm{d} \pi^{\perp} \\
\gamma \pi^{\circ} & -\mathrm{d} \pi^{\circ}
\end{array}\right],
$$

$\pi^{\perp}, \pi^{\circ}$ being defined in (2.10) and (2.11), respectively, and $\gamma$ a positive parameter. Then, there exist positive constants $C_{1}, C_{1}^{\prime}$ that depend only on the Poincaré constant $C_{\mathrm{P}}$ and on the parameter $\alpha$, such that

$$
\begin{gathered}
\left\langle T\left[\begin{array}{l}
u \\
p
\end{array}\right],\left[\begin{array}{l}
v \\
q
\end{array}\right]\right\rangle_{V_{k}^{\prime}, V_{k}} \geqslant C_{1}\left\|\left[\begin{array}{l}
u \\
p
\end{array}\right]\right\|_{V_{k}}^{2}=C_{1}\left(\|u\|_{H \Lambda^{k}}^{2}+\|p\|_{H \Lambda^{k-1}}^{2}\right), \\
\left\|\left[\begin{array}{l}
v \\
q
\end{array}\right]\right\|_{V_{k}} \leqslant C_{1}^{\prime}\left\|\left[\begin{array}{l}
u \\
p
\end{array}\right]\right\|_{V_{k}} .
\end{gathered}
$$

The same result holds with $\alpha=0$ provided that $F_{2}$ corresponds to $f_{2} \in \delta H_{\Gamma_{\mathrm{D}}} \Lambda^{k}(D)$.

The well-posedness of problem (2.21) is proved in Arnold et al. (2006) by showing that the bounded bilinear and symmetric form $\langle T \cdot, \cdot\rangle: V_{k} \times V_{k} \rightarrow \mathbb{R}$ satisfies the inf-sup condition (2.23), (2.24) (see Babuška \& Aziz, 1972; Brezzi \& Fortin, 1991). However, we report it entirely (with a slightly different choice of test functions) as a preparatory step for the proofs we will propose later on. 
Proof. We need to show (2.23) and (2.24). Let us start by considering $\alpha>0$. For a given $\left[\begin{array}{l}u \\ p\end{array}\right]$ we use the Hodge decomposition (2.9):

$$
\left[\begin{array}{l}
u \\
p
\end{array}\right]=\left[\begin{array}{l}
\mathrm{d} u^{\circ}+u^{\perp} \\
\mathrm{d} p^{\circ}+p^{\perp}
\end{array}\right],
$$

with $\mathrm{d} u^{\circ} \in \mathfrak{B}_{k}, \mathrm{~d} p^{\circ} \in \mathfrak{B}_{k-1}, u^{\perp} \in \mathfrak{B}_{k}^{\perp}$ and $p^{\perp} \in \mathfrak{B}_{k-1}^{\perp}$. As test functions, we choose

$$
\left[\begin{array}{l}
v \\
q
\end{array}\right]=P\left[\begin{array}{l}
\mathrm{d} u^{\circ}+u^{\perp} \\
\mathrm{d} p^{\circ}+p^{\perp}
\end{array}\right]=\left[\begin{array}{c}
u^{\perp}+\mathrm{d} p^{\perp} \\
\gamma u^{\circ}-\mathrm{d} p^{\circ}
\end{array}\right],
$$

where $\gamma$ is a positive parameter to be set later. Substituting (2.25) into (2.23), using the property $\mathrm{d} \circ \mathrm{d}=$ 0 , the Hodge decomposition (2.9) and the Poincaré inequality (2.12), we find

$$
\begin{aligned}
\left\langle T\left[\begin{array}{l}
u \\
p
\end{array}\right],\left[\begin{array}{l}
v \\
q
\end{array}\right]\right\rangle_{V_{k}^{\prime}, V_{k}}= & (\mathrm{d} u, \mathrm{~d} v)+(v, \mathrm{~d} p)+(u, \mathrm{~d} q)-\alpha(p, q) \\
= & \left\|\mathrm{d} u^{\perp}\right\|^{2}+\left\|\mathrm{d} p^{\perp}\right\|^{2}+\gamma\left\|\mathrm{d} u^{\circ}\right\|^{2}+\alpha\left\|\mathrm{d} p^{\circ}\right\|^{2}-\alpha \gamma\left(p^{\perp}, u^{\circ}\right) \\
\geqslant & \left\|\mathrm{d} u^{\perp}\right\|^{2}+\left\|\mathrm{d} p^{\perp}\right\|^{2}+\gamma\left\|\mathrm{d} u^{\circ}\right\|^{2}+\alpha\left\|\mathrm{d} p^{\circ}\right\|^{2} \\
& -\frac{\alpha \gamma^{1 / 2}}{2}\left(C_{\mathrm{P}}^{2}\left\|\mathrm{~d} p^{\perp}\right\|^{2}+\gamma C_{\mathrm{P}}^{2}\left\|\mathrm{~d} u^{\circ}\right\|^{2}\right) \\
\geqslant & \left\|\mathrm{d} u^{\perp}\right\|^{2}+\left(1-\frac{\alpha}{2} \gamma^{1 / 2} C_{\mathrm{P}}^{2}\right)\left\|\mathrm{d} p^{\perp}\right\|^{2} \\
& +\gamma\left(1-\frac{\alpha \gamma^{1 / 2} C_{\mathrm{P}}^{2}}{2}\right)\left\|\mathrm{d} u^{\circ}\right\|^{2}+\alpha\left\|\mathrm{d} p^{\circ}\right\|^{2} .
\end{aligned}
$$

It is possible to choose $\gamma$ in order to make (2.23) true with $C_{1}=C_{1}\left(C_{\mathrm{P}}, \alpha\right)$. The inequality (2.24) with $C_{1}=C_{1}^{\prime}\left(C_{\mathrm{P}}, \alpha\right)$ follows from the Hodge decomposition (2.9) and Poincaré inequality (2.12).

The proof in the case $\alpha=0$ is very similar. Suppose $f_{2} \in \delta H_{\Gamma_{\mathrm{D}}} \Lambda^{k}(D)$. In order to have a unique solution, we need to look for $p \in \mathfrak{B}_{k-1}^{\perp}$. With fixed $u=\mathrm{d} u^{\circ}+u^{\perp} \in H_{\Gamma_{\mathrm{D}}} \Lambda^{k}(D)$ we again choose the test functions as in (2.25): $v=\mathrm{d} p+u^{\perp} \in H_{\Gamma_{\mathrm{D}}} \Lambda^{k}(D)$ and $q=u^{\circ} \in \mathfrak{B}_{k-1}^{\perp}$. Using the Poincaré inequality (2.12) and the orthogonal decomposition (2.9) we are able to prove the relations (2.23) and (2.24).

A simple consequence of Theorem 2.6 (see Brezzi \& Fortin, 1991) is that there exists a positive constant $K=K\left(C_{\mathrm{P}}, \alpha\right)$ such that

$$
\left\|\left[\begin{array}{l}
u \\
p
\end{array}\right]\right\|_{V_{k}} \leqslant K\left\|\left[\begin{array}{l}
F_{1} \\
F_{2}
\end{array}\right]\right\|_{V_{k}^{\prime}} .
$$

2.2.1 Translation to the language of PDEs Let us consider the case $D \subset \mathbb{R}^{3}$, naturally identifying the tangent space at each point $x \in D$ with $\mathbb{R}^{3}$. Owing to the identification of $\mathrm{Alt}^{0} \mathbb{R}^{3}$ and $\mathrm{Alt}^{3} \mathbb{R}^{3}$ with $\mathbb{R}$, and of $\mathrm{Alt}^{1} \mathbb{R}^{3}$ and $\mathrm{Alt}^{2} \mathbb{R}^{3}$ with $\mathbb{R}^{3}$, we can establish correspondences between the spaces of differential forms and scalar or vector fields. These fields are called proxy fields. In particular, we can identify each 0 -form and 3-form with a scalar-valued function, and each 1-form and 2-form with a vector-valued 
TABLE 1 Correspondences in terms of proxy fields between the space of differential forms $H \Lambda^{k}(D)$ and the classical spaces of functions and vector fields, in the case $n=3$

\begin{tabular}{lccc}
\hline & $H_{\Gamma_{\mathrm{D}}} \Lambda^{k}(D)$ & $\mathrm{d}$ & $\left.\operatorname{Tr}\right|_{\Gamma_{\mathrm{D}}} u$ \\
\hline$k=0$ & $H_{\Gamma_{\mathrm{D}}}^{1}(D)$ & $\nabla$ & $\left.u\right|_{\Gamma_{\mathrm{D}}}$ \\
$k=1$ & $H_{\Gamma_{\mathrm{D}}}(\operatorname{curl}, D)$ & $\operatorname{curl}$ & $u \times\left. n\right|_{\Gamma_{\mathrm{D}}}$ \\
$k=2$ & $H_{\Gamma_{\mathrm{D}}}(\operatorname{div}, D)$ & $\operatorname{div}$ & $\left.u \cdot n\right|_{\Gamma_{\mathrm{D}}}$ \\
$k=3$ & $L^{2}(D)$ & 0 & 0 \\
\hline
\end{tabular}

function. Table 1 summarizes the correspondences in terms of proxy fields for the spaces of differential forms $H_{\Gamma_{\mathrm{D}}} \Lambda^{k}(D)$, the exterior derivative operators and the trace operators. Based on the identifications in Table 1 we can reinterpret the de Rham complex (2.7) as follows:

$$
0 \rightarrow H_{\Gamma_{\mathrm{D}}}^{1}(D) \stackrel{\nabla}{\rightarrow} H_{\Gamma_{\mathrm{D}}}(\operatorname{curl}, D) \stackrel{\text { curl }}{\longrightarrow} H_{\Gamma_{\mathrm{D}}}(\operatorname{div}, D) \stackrel{\text { div }}{\longrightarrow} L^{2}(D) \rightarrow 0
$$

where $H_{\Gamma_{\mathrm{D}}}^{1}(D), H_{\Gamma_{\mathrm{D}}}(\operatorname{curl}, D), H_{\Gamma_{\mathrm{D}}}(\operatorname{div}, D)$ denote the classical Sobolev spaces of functions in $H^{1}(D)$, $H(\operatorname{curl}, D), H(\operatorname{div}, D)$, respectively, with trace vanishing on $\Gamma_{\mathrm{D}}$. In this section, we will use the symbol $(\cdot, \cdot)$ to denote the inner product in $L^{2}(D)$ that corresponds by proxy identifications to the inner product in $L^{2} \Lambda^{k}(D)$.

- Let us start with $k=0$. In this case $H_{\Gamma_{\mathrm{D}}} \Lambda^{-1}(D)=0$, so $p=0$. Then, $u \in H_{\Gamma_{\mathrm{D}}}^{1}(D)$ satisfies

$$
(\nabla u, \nabla v)=\left(f_{1}, v\right) \quad \forall v \in H_{\Gamma_{\mathrm{D}}}^{1}(D)
$$

We obtain the usual weak formulation of the Poisson equation equipped with homogeneous Dirichlet boundary conditions on $\Gamma_{\mathrm{D}}$ and homogeneous Neumann boundary conditions on $\Gamma_{\mathrm{N}}$.

- For $k=1$ and $\alpha=0$, the linear operator $T$ of order 2 defined in (2.16) is represented by the matrix

$$
T=\left[\begin{array}{cc}
\operatorname{curl}^{2} & \nabla \\
-\operatorname{div} & 0
\end{array}\right]
$$

Problem (2.21) is the weak formulation of the magnetostatic/electrostatic equations (see e.g. Bossavit, 1998; Hiptmair, 2002; Monk, 2003). Indeed, $V_{1}=\left[\begin{array}{c}H_{\Gamma_{\mathrm{D}}}(\mathrm{curl}, D) \\ H_{\Gamma_{\mathrm{D}}}^{1}(D)\end{array}\right]$ and $\left[\begin{array}{l}u \\ p\end{array}\right] \in V_{1}$ satisfies

$$
\left\{\begin{array}{l}
(\operatorname{curl} u, \operatorname{curl} v)+(\nabla p, v)=\left(f_{1}, v\right), \quad \forall\left[\begin{array}{l}
v \\
q
\end{array}\right] \in V_{1} .
\end{array}\right.
$$

- When $k=2$,

$$
T=\left[\begin{array}{cc}
-\nabla \operatorname{div} & \text { curl } \\
\operatorname{curl} & -\alpha \mathrm{Id}
\end{array}\right]
$$


Problem (2.21) is the mixed formulation of the vectorial Poisson equation: find $\left[\begin{array}{l}u \\ p\end{array}\right] \in V_{2}=$ $\left[\begin{array}{c}H_{\Gamma_{\mathrm{D}}}(\operatorname{div}, D) \\ H_{\Gamma_{\mathrm{D}}}(\operatorname{curl}, D)\end{array}\right]$ such that

$$
\left\{\begin{array}{l}
(\operatorname{div} u, \operatorname{div} v)+(\operatorname{curl} p, v)=\left(f_{1}, v\right), \\
(u, \operatorname{curl} q)-\alpha(p, q)=\left(f_{2}, q\right),
\end{array} \quad \forall\left[\begin{array}{l}
v \\
q
\end{array}\right] \in V_{2} .\right.
$$

- Finally, for $k=3$, problem (2.21) models flow in porous media. We can reinterpret $T$, the linear tensor operator of order 2 , as

$$
T=\left[\begin{array}{cc}
0 & \operatorname{div} \\
-\nabla & -\alpha \mathrm{Id}
\end{array}\right]
$$

where $\alpha>0$ is linked to the inverse of the permeability. Hence, problem (2.21) is the Darcy equations: find $\left[\begin{array}{l}u \\ p\end{array}\right] \in V_{3}=\left[\begin{array}{c}L^{2}(D) \\ H_{\Gamma_{\mathrm{D}}}(\operatorname{div}, D)\end{array}\right]$ such that

$$
\left\{\begin{array}{l}
(\operatorname{div} p, v)=\left(f_{1}, v\right), \\
(u, \operatorname{div} q)-\alpha(p, q)=0,
\end{array} \quad \forall\left[\begin{array}{l}
v \\
q
\end{array}\right] \in V_{3} .\right.
$$

\section{Stochastic Sobolev spaces of differential forms and the stochastic Hodge Laplacian}

Let $(\Omega, \mathcal{A}, \mathbb{P})$ be a complete probability space and $V$ be a separable Hilbert space. We define the stochastic counterpart of $V$ as the Hilbert space given by the tensor product $V \otimes L^{2}(\Omega, \mathrm{d} \mathbb{P})$, where $L^{m}(\Omega, \mathrm{d} \mathbb{P})$ is the standard Lebesgue space of functions whose $m$ th power is integrable with respect to the probability measure.

Let $L^{2}(\Omega ; V)$ be the Bochner space composed of functions $u$ such that $\omega \mapsto\|u(\omega)\|_{V}^{2}$ is measurable and integrable, so that $\|u\|_{L^{2}(\Omega ; V)}:=\left(\int_{\Omega}\|u(\omega)\|_{V}^{2} \mathrm{~d} \mathbb{P}(\omega)\right)^{1 / 2}$ is finite. We observe that there is a unique isomorphism from $V \otimes L^{2}(\Omega, \mathrm{d} \mathbb{P})$ to $L^{2}(\Omega ; V)$ which maps $\psi \otimes \mu \in V \otimes L^{2}(\Omega, \mathrm{d} \mathbb{P})$ onto the function $\omega \mapsto \mu(\omega) \psi \in V$. The definition of the Hilbert space $L^{2}(\Omega ; V)$ easily generalizes to the space $L^{m}(\Omega ; V)$ with $m \geqslant 1$. We say that a random field $u: \Omega \rightarrow V$ is in the Bochner space $L^{m}(\Omega ; V)$ if $\omega \mapsto\|u(\omega)\|_{V}^{m}$ is measurable and integrable, so that $\|u\|_{L^{m}(\Omega ; V)}:=\left(\int_{\Omega}\|u(\omega)\|_{V}^{m} \mathrm{~d} \mathbb{P}(\omega)\right)^{1 / m}$ is finite.

Let $\left[\begin{array}{l}F_{1} \\ F_{2}\end{array}\right] \in L^{m}\left(\Omega ; V_{k}^{\prime}\right)$, with $m \geqslant 1$, defined as the stochastic version of (2.19) and (2.20), be given:

$$
\begin{gathered}
F_{1}(\omega): H_{\Gamma_{\mathrm{D}}} \Lambda^{k}(D) \rightarrow \mathbb{R}, \quad F_{1}(\omega)(v):=\left(f_{1}(\omega), v\right), \\
F_{2}(\omega): H_{\Gamma_{\mathrm{D}}} \Lambda^{k-1}(D) \rightarrow \mathbb{R}, \quad F_{2}(\omega)(q):=\left(f_{2}(\omega), q\right),
\end{gathered}
$$

where $\left[\begin{array}{l}f_{1} \\ f_{2}\end{array}\right] \in L^{m}\left(\Omega ; W_{k}\right)$ is also given. The stochastic counterpart of problem $(2.21)$ is as follows.

\section{Stochastic Problem}

$$
\begin{gathered}
\text { Given } m \geqslant 1 \text { and }\left[\begin{array}{l}
F_{1} \\
F_{2}
\end{array}\right] \in L^{m}\left(\Omega ; V_{k}^{\prime}\right), \quad \text { find }\left[\begin{array}{l}
u \\
p
\end{array}\right] \in L^{m}\left(\Omega ; V_{k}\right) \text { such that } \\
T\left[\begin{array}{l}
u(\omega) \\
p(\omega)
\end{array}\right]=\left[\begin{array}{l}
F_{1}(\omega) \\
F_{2}(\omega)
\end{array}\right] \quad \text { in } V_{k}^{\prime} \text {, a.e. in } \Omega \text {. }
\end{gathered}
$$


TheOREm 3.1 (Well-posedness of the stochastic Hodge Laplacian) For every $\alpha>0$ problem (3.1) is well posed, so that there exists a unique solution that depends continuously on the data. The same result holds with $\alpha=0$, provided that $F_{2}$ corresponds to $f_{2} \in L^{m}\left(\Omega ; \delta H_{\Gamma_{\mathrm{D}}} \Lambda^{k}(D)\right)$.

Proof. The result follows by the well-posedness of the deterministic Hodge Laplacian for a.e. $\omega \in \Omega$ (Theorem 2.6), and using the fact that $\left[\begin{array}{l}F_{1} \\ F_{2}\end{array}\right] \in L^{m}\left(\Omega ; V_{k}^{\prime}\right)$ and (2.26). Observe that the constant $K$ in (2.26) does not depend on $\omega$.

\section{Deterministic problems for the statistics of $u$ and $p$}

We are interested in the statistical moments of the unique stochastic solution $\left[\begin{array}{l}u \\ p\end{array}\right]$ of the stochastic problem (3.1). We exploit the linearity of the system $T\left[\begin{array}{l}u(\omega) \\ p(\omega)\end{array}\right]=\left[\begin{array}{l}F_{1}(\omega) \\ F_{2}(\omega)\end{array}\right]$ to derive the moment equations, that is, the deterministic equations solved by the statistical moments of the unique stochastic solution $\left[\begin{array}{l}u \\ p\end{array}\right]$. The main achievement is the constructive proof of the inf-sup condition for the tensor product operator $T^{\otimes m}$ stated in Theorem 4.6, equivalent to the well-posedness of the $m$ th moment problem. Indeed, this proof extends to the case of sparse tensor product approximations (see Section 6.3).

\subsection{Tensor product of operators on Hilbert spaces}

Given $T_{1}: V_{1} \rightarrow V_{1}^{\prime}, T_{2}: V_{2} \rightarrow V_{2}^{\prime}$, continuous operators on the Hilbert spaces $V_{1}$ and $V_{2}$, respectively, then the tensor product operator $T_{1} \otimes T_{2}: V_{1} \otimes V_{2} \rightarrow V_{1}^{\prime} \otimes V_{2}^{\prime}$ is defined on functions of the type $\phi \otimes \psi$, with $\phi \in V_{1}, \psi \in V_{2}$ as

$$
\left(T_{1} \otimes T_{2}\right)(\phi \otimes \psi)=T_{1} \phi \otimes T_{2} \psi \in V_{1}^{\prime} \otimes V_{2}^{\prime},
$$

and then extended by linearity and density (see Reed \& Simon, 1980 and the references therein). The tensor product of two bounded operators on Hilbert space is still a bounded operator, as stated by the following proposition.

Proposition 4.1 Let $T_{1}: V_{1} \rightarrow V_{1}^{\prime}, T_{2}: V_{2} \rightarrow V_{2}^{\prime}$ be bounded operators on Hilbert spaces $V_{1}$ and $V_{2}$, respectively. Then

$$
\left\|T_{1} \otimes T_{2}\right\|_{\mathcal{L}\left(V_{1} \otimes V_{2}, V_{1}^{\prime} \otimes V_{2}^{\prime}\right)}=\left\|T_{1}\right\|_{\mathcal{L}\left(V_{1}, V_{1}^{\prime}\right)}\left\|T_{2}\right\|_{\mathcal{L}\left(V_{2}, V_{2}^{\prime}\right)}
$$

Proof. For the proof, see Reed \& Simon (1980).

The definition of the tensor product of two operators on Hilbert spaces and Proposition 4.1 generalize to a tensor product of any finite number of operators defined on Hilbert spaces.

We detail now the vector case, since it will be useful in the next section. Let $V_{1}=V_{2}=V_{k}$, where $V_{k}$ is defined in (2.14), and $T_{1}=T_{2}=T$, where $T=(T)_{i, j=1,2}: V_{k} \rightarrow V_{k}^{\prime}$ is the linear operator of order 2 defined in (2.16). The tensor product operator $T^{\otimes m}:=\underbrace{T \otimes \cdots \otimes T}_{m},(m \geqslant 1$ integer $)$, is the operator of order $2 m$ that maps tensors in $V_{k}^{\otimes m}$ to tensors is $\left(V_{k}^{\prime}\right)^{\otimes m}$ defined as

$$
\left(T^{\otimes m}\right)_{i_{1} \ldots i_{2 m}}=T_{i_{1} i_{2}} \otimes \cdots \otimes T_{i_{2 m-1} i_{2 m}} .
$$

Given $X \in V_{k}^{\otimes m}, T^{\otimes m} X$ is a tensor of order $m$ in $\left(V_{k}^{\prime}\right)^{\otimes m}$ given by

$$
\left(T^{\otimes m} X\right)_{i_{1} \ldots i_{m}}=\sum_{j_{1}, \ldots, j_{m}=1}^{2}\left(T_{i_{1} j_{1}} \otimes \cdots \otimes T_{i_{m} j_{m}}\right) X_{j_{1} \ldots j_{m}}, \quad i_{1}, \ldots, i_{m}=1,2 .
$$


Definition 4.2 Let $T$ and $V_{k}$ be as before and let $X \in V_{k}^{\otimes m}$ and $Y \in V_{k}^{\otimes m}$. We define

$$
\left\langle T^{\otimes m} X, Y\right\rangle=\sum_{i_{1}, \ldots, i_{m}=1}^{2} \sum_{j_{1}, \ldots, j_{m}=1}^{2}\left\langle T_{i_{1}, j_{1}} \cdots T_{i_{m}, j_{m}} X_{j_{1}, \ldots, j_{m}}, Y_{i_{1}, \ldots, i_{m}}\right\rangle .
$$

\subsection{Equations for the mth moment}

Let $v \in L^{m}(\Omega ; V), m \geqslant 1$ integer, where $V$ is a Hilbert space and $L^{m}(\Omega ; V)$ is defined as in Section 3 . Then $v^{\otimes m}:=\underbrace{v \otimes \cdots \otimes v}_{m \text { times }} \in L^{1}\left(\Omega, V^{\otimes m}\right)$, where from now on $V^{\otimes m}$ denotes the tensor product space $\underbrace{V \otimes \cdots \otimes V}_{m \text { times }}$. The $m$ th moment of $v$ is defined as

$$
\mathcal{M}^{m}[v]:=\mathbb{E}[v \otimes \cdots \otimes v]=\int_{\Omega} v(\omega) \otimes \cdots \otimes v(\omega) \mathrm{d} \mathbb{P}(\omega) \in V^{\otimes m}
$$

It clearly holds that $\left\|\mathcal{M}^{m}[v]\right\|_{V^{\otimes m}} \leqslant\|v\|_{L^{m}(\Omega ; V)}^{m}$. The definition (4.4) with $m=1$ gives the expected value of $v, \mathbb{E}[v]$. Moreover, definition (4.4) easily generalizes to the vector case.

Following von Petersdorff \& Schwab (2006), we analyse the $m$ th moment equation for $m \geqslant 1$. Suppose $\left[\begin{array}{l}F_{1} \\ F_{2}\end{array}\right] \in L^{m}\left(\Omega ; V_{k}^{\prime}\right)$, so that $\left[\begin{array}{l}u \\ p\end{array}\right] \in L^{m}\left(\Omega ; V_{k}\right)$. To derive the deterministic $m$ th moment problem we tensorize the stochastic problem (3.1) with itself $m$ times:

$$
\underbrace{T \otimes \cdots \otimes T}_{m \text { times }}\left[\begin{array}{l}
u(\omega) \\
p(\omega)
\end{array}\right]^{\otimes m}=\left[\begin{array}{l}
F_{1}(\omega) \\
F_{2}(\omega)
\end{array}\right]^{\otimes m} \quad \text { in }\left(V_{k}^{\prime}\right)^{\otimes m}, \text { for a.e. } \omega \in \Omega .
$$

We take the expectation on both sides and we exploit the commutativity between the operators $T$ and $\mathbb{E}$. By definition, $\mathbb{E}\left[\begin{array}{l}u \\ p\end{array}\right]^{\otimes m}=\mathcal{M}^{m}\left[\begin{array}{l}u \\ p\end{array}\right]$. Thus, $\mathcal{M}^{m}\left[\begin{array}{l}u \\ p\end{array}\right]$ is a solution of the following.

\section{$m$-Points Correlation Problem}

$$
\begin{gathered}
\text { Given } m \geqslant 1 \text { integer and }\left[\begin{array}{l}
F_{1} \\
F_{2}
\end{array}\right] \in L^{m}\left(\Omega ; V_{k}^{\prime}\right) \text {, find } M_{s}^{\otimes m} \in V_{k}^{\otimes m} \text { such that } \\
T^{\otimes m} M_{s}^{\otimes m}=\mathcal{M}^{m}\left[\begin{array}{l}
F_{1} \\
F_{2}
\end{array}\right] \text { in }\left(V_{k}^{\prime}\right)^{\otimes m} .
\end{gathered}
$$

Here, $\mathcal{M}^{m}\left[\begin{array}{l}F_{1} \\ F_{2}\end{array}\right] \in\left(V_{k}^{\prime}\right)^{\otimes m}$ is defined as

$$
\mathcal{M}^{m}\left[\begin{array}{l}
F_{1} \\
F_{2}
\end{array}\right]\left(\left[\begin{array}{l}
v \\
q
\end{array}\right]\right):=\left(\mathcal{M}^{m}\left[\begin{array}{l}
f_{1} \\
f_{2}
\end{array}\right],\left[\begin{array}{l}
v \\
q
\end{array}\right]\right)_{W_{k}^{\otimes m}} \quad \forall\left[\begin{array}{l}
v \\
q
\end{array}\right] \in V_{k}^{\otimes m} .
$$

We note that in the right-hand side of (4.5) we have the $m$-points correlation of the loading terms of problem (3.1). 
REMARK 4.3 Note that the first moment problem is a saddle-point problem and (4.5) is composed of $m$ 'nested' saddle-point problems. Indeed, if for example $m=2$, then $T \otimes T$ can be represented by the matrix

$$
T \otimes T=\left[\begin{array}{cc|cc}
\delta \mathrm{d} \otimes \delta \mathrm{d} & \delta \mathrm{d} \otimes \mathrm{d} & \mathrm{d} \otimes \delta \mathrm{d} & \mathrm{d} \otimes \mathrm{d} \\
\delta \mathrm{d} \otimes \delta & \delta \mathrm{d} \otimes-\alpha \mathrm{Id} & \mathrm{d} \otimes \delta & \mathrm{d} \otimes-\alpha \mathrm{Id} \\
\hline \delta \otimes \delta \mathrm{d} & \delta \otimes \mathrm{d} & -\alpha \mathrm{Id} \otimes \delta \mathrm{d} & -\alpha \mathrm{Id} \otimes \mathrm{d} \\
\delta \otimes \delta & \delta \otimes-\alpha \mathrm{Id} & -\alpha \mathrm{Id} \otimes \delta & -\alpha \mathrm{Id} \otimes-\alpha \mathrm{Id}
\end{array}\right] .
$$

TheOREm 4.4 (Well-posedness of the $m$ th problem) For every $\alpha>0$, problem (4.5) is well posed, so that there exists a unique solution that depends continuously on the data. The same result holds with $\alpha=0$, provided that $F_{2}$ corresponds to $f_{2} \in L^{m}\left(\Omega ; \delta H_{\Gamma_{\mathrm{D}}} \Lambda^{k}(D)\right)$.

Proof. In the case $m=1$, the theorem follows directly from the well-posedness of the deterministic Hodge Laplacian. Suppose $m \geqslant 2$. Theorem 4.4 can be proved by a simple tensor product argument, as follows. Since problem (2.21) is well posed, the inverse operator $T^{-1}$ exists and is linear and bounded. Now, we consider the tensor operator $\left(T^{-1}\right)^{\otimes m}=\underbrace{T^{-1} \otimes \cdots \otimes T^{-1}}_{m \text { times }}$. It is the inverse operator of $T^{\otimes m}$. Moreover, it is linear and bounded (Proposition 4.1). Hence, we can immediately conclude the wellposedness of problem (4.5).

REMARK 4.5 The approach presented in the proof is not completely satisfactory in view of a finitedimensional approximation. Indeed, when considering a finite-dimensional version of the operator, $T_{h}:=\left.T\right|_{V_{k, h}}: V_{k, h} \rightarrow V_{k, h}^{\prime}$, where $V_{k, h}$ is a finite-dimensional subspace of $V_{k}$, and aiming at proving the well-posedness of the tensor operator $\left(T_{h}\right)^{\otimes m}=\underbrace{T_{h} \otimes \cdots \otimes T_{h}}_{m \text { times }}$, this tensor product argument applies only if the finite-dimensional subspace is a tensor product space $V_{k, h}^{\otimes m}$. It will not apply straightforwardly if sparse tensor product spaces are considered instead.

\subsection{Constructive proof of the inf-sup condition for the tensorized problem}

Here, we propose an alternative proof of Theorem 4.4 that consists in showing the inf-sup condition for $T^{\otimes m}, m \geqslant 2$ integer. This proof will be used later on to prove the stability of an STP-FE discretization, which is of practical interest for moderately large $m$ as it reduces considerably the curse of dimensionality with respect to an FTP approximation.

A result equivalent to Theorem 4.4 is the following theorem.

THeOREM 4.6 (Tensorial inf-sup condition) For every $M_{s}^{\otimes m} \in V_{k}^{\otimes m}$, take $M_{t}^{\otimes m}=P^{\otimes m} M_{s}^{\otimes m} \in V_{k}^{\otimes m}$, where $P$ is defined in (2.22). Then, there exist positive constants

$$
C_{m}=C_{m}\left(\alpha, C_{\mathrm{P}, 1},\|T\|_{\mathcal{L}\left(V_{k}, V_{k}^{\prime}\right)},\|P\|_{\mathcal{L}\left(V_{k}, V_{k}\right)}\right), \quad C_{m}^{\prime}=C_{m}^{\prime}\left(\alpha, C_{P, 1},\|T\|_{\mathcal{L}\left(V_{k}, V_{k}^{\prime}\right)},\|P\|_{\mathcal{L}\left(V_{k}, V_{k}\right)}\right)
$$

such that

$$
\begin{aligned}
\left.\left\langle T^{\otimes m} M_{s}^{\otimes m}, M_{t}^{\otimes m}\right\rangle_{\left(V_{k}^{\prime}\right)}\right)^{\otimes m}, V_{k}^{\otimes m} & \geqslant C_{m}\left\|M_{s}^{\otimes m}\right\|_{V_{k}^{\otimes m}}^{2}, \\
\left\|M_{t}^{\otimes m}\right\|_{V_{k}^{\otimes m}} & \leqslant C_{m}^{\prime}\left\|M_{s}^{\otimes m}\right\|_{V_{k}^{\otimes m}},
\end{aligned}
$$

where $C_{\mathrm{P}, 1}$ is the tensorial Poincaré constant (see Lemma 4.7). 
Before presenting the proof we state the tensorized versions of the Hodge decomposition and the Poincaré inequality, which are two key ingredients in the proof of the inf-sup condition for the deterministic problem (2.21).

Let us write the space $V_{k}^{\otimes m}$ as

$$
V_{k}^{\otimes m}=V_{k} \otimes V_{k}^{\otimes(m-1)}=\left[\begin{array}{c}
H_{\Gamma_{\mathrm{D}}} \Lambda^{k}(D) \\
H_{\Gamma_{\mathrm{D}}} \Lambda^{k-1}(D)
\end{array}\right] \otimes V_{k}^{\otimes(m-1)}=\left[\begin{array}{c}
U_{k}^{m} \\
U_{k-1}^{m}
\end{array}\right],
$$

where we define

$$
\begin{aligned}
U_{k}^{m} & :=H_{\Gamma_{\mathrm{D}}} \Lambda^{k}(D) \otimes V_{k}^{\otimes(m-1)}, \\
U_{k-1}^{m} & :=H_{\Gamma_{\mathrm{D}}} \Lambda^{k-1}(D) \otimes V_{k}^{\otimes(m-1)} .
\end{aligned}
$$

We obtain the tensorial Hodge decomposition following the idea of the one-dimensional Hodge decomposition (2.9). Indeed, for every integer $m \geqslant 2$, we split $U_{k}^{m}\left(U_{k-1}^{m}\right.$ is analogous) as follows.

\section{Tensorial Hodge Decomposition:}

$$
U_{k}^{m}=\mathfrak{B}_{k}^{m} \oplus \mathfrak{B}_{k}^{m, \perp},
$$

where

$$
\begin{aligned}
\mathfrak{B}_{k}^{m} & :=\mathrm{d} \otimes \mathrm{Id}^{\otimes(m-1)} U_{k-1}^{m}=\mathfrak{B}_{k} \otimes V_{k}^{\otimes(m-1)}, \\
\mathfrak{B}_{k}^{m, \perp} & :=\mathfrak{B}_{k}^{\perp} \otimes V_{k}^{\otimes(m-1)}
\end{aligned}
$$

and $\mathfrak{B}_{k}, \mathfrak{B}_{k}^{\perp}$ are defined as in Section 2. The tensor operators $\pi^{\perp} \otimes \mathrm{Id}^{\otimes(m-1)}$ and $\pi^{\circ} \otimes \mathrm{Id}^{\otimes(m-1)}$, where $\pi^{\perp}$ and $\pi^{\circ}$ are defined in (2.10) and (2.11), respectively, act on $U_{k}^{m}$ ( $U_{k-1}^{m}$ is analogous) as follows:

$$
\begin{gathered}
\pi^{\perp} \otimes \mathrm{Id}^{\otimes(m-1)}: U_{k}^{m}=\mathfrak{B}_{k}^{m} \oplus \mathfrak{B}_{k}^{m, \perp} \rightarrow \mathfrak{B}_{k}^{m, \perp}, \\
v=\mathrm{d} \otimes \mathrm{Id}^{\otimes(m-1)} v^{\circ}+v^{\perp} \mapsto v^{\perp}, \\
\pi^{\circ} \otimes \mathrm{Id}^{\otimes((m-1)}: U_{k}^{m}=\mathfrak{B}_{k}^{m} \oplus \mathfrak{B}_{k}^{m, \perp} \rightarrow \mathfrak{B}_{k-1}^{m, \perp}, \\
v=\mathrm{d} \otimes \mathrm{Id}^{\otimes(m-1)} v^{\circ}+v^{\perp} \mapsto v^{\circ} .
\end{gathered}
$$

The tensorial Poincaré inequality is proved in the following lemma.

Lemma 4.7 (Tensorial Poincaré inequality) For every integer $m \geqslant 2$, there exists a positive constant $C_{P, 1}$ such that

$$
\|v\|_{\left(L^{2} \Lambda^{k}\right) \otimes m} \leqslant C_{P, 1}\|\mathrm{Id} \otimes \cdots \otimes \underbrace{\mathrm{d}}_{i} \otimes \cdots \otimes \operatorname{Id} v\|_{L^{2} \Lambda^{k} \otimes \cdots \otimes} \underbrace{L^{2} \Lambda^{k}}_{i} \otimes \cdots \otimes L^{2} \Lambda^{k}
$$

for all $v \in L^{2} \Lambda^{k}(D) \otimes \cdots \otimes \underbrace{\left(\mathfrak{Z}_{k}^{\perp}\right)}_{i} \otimes \cdots \otimes L^{2} \Lambda^{k}(D)$, where $\mathfrak{Z}_{k}^{\perp}$ is defined in Section 2.1. 
Proof. We know that $H \Lambda^{k}(D)$ is a Hilbert space with the inner product $(u, v)_{H \Lambda^{k}}$ and $(u, u)_{H \Lambda^{k}}=$ $\|u\|_{H \Lambda^{k}}^{2}$. Besides, we know that $\mathfrak{Z}_{k}^{\perp}$ is a Hilbert space with the equivalent inner product $(\mathrm{d} u, \mathrm{~d} v)$ and norm $\|\mathrm{d} u\|=(\mathrm{d} u, \mathrm{~d} u)$. A consequence of the open mapping theorem states that, given $m$ Hilbert spaces $H_{1}, \ldots, H_{m}$, the topology of $H_{1} \otimes \cdots \otimes H_{m}$ depends only on the topology and not on the choice of the inner products of $H_{1}, \ldots, H_{m}$. If we apply this statement with $H_{i}=\mathfrak{Z}_{k}^{\perp}$ and $H_{j}=H \Lambda^{k}(D), i \neq j$, we can conclude the inequality (4.15).

A simple consequence of the previous lemma is

$$
\|v\|_{\left(L^{2} \Lambda^{k}\right)^{\otimes m}} \leqslant C_{\mathrm{P}, m}\left\|\mathrm{~d}^{\otimes m} v\right\|_{\left(L^{2} \Lambda^{k+1}\right) \otimes m} \quad \forall v \in\left(\mathfrak{Z}_{k}^{\perp}\right)^{\otimes m}
$$

where $C_{\mathrm{P}, m}>0$ depends only on the domain $D$ and on $m$.

Proof of Theorem 4.6. As shown before, $\mathcal{M}^{m}\left[\begin{array}{l}u \\ p\end{array}\right]$ is a solution of (4.5). Uniqueness of the solution of problem (4.5) is related to the global inf-sup condition (4.7), (4.8) (see Babuška \& Aziz, 1972; Brezzi $\&$ Fortin, 1991). Suppose $\alpha>0$ (the case $\alpha=0$ is analogous). To lighten the notation, in the proof we use the brackets $\langle\cdot, \cdot\rangle$ without specifying the spaces we consider, when no ambiguity arises. We use the tensorial Hodge decomposition (4.12) and the tensorial Poincaré inequality (Lemma 4.7). We prove (4.7) by induction. In Theorem 2.6, we have already proved the inf-sup condition with $m=1$. Now, suppose $m=2$. We fix $M_{s}^{\otimes 2}=\left[\begin{array}{c}\left(M_{s}^{\otimes 2}\right)_{1:} \\ \left(M_{s}^{\otimes 2}\right)_{2:}\end{array}\right]$ where $\left(M_{s}^{\otimes 2}\right)_{1:}$ (respectively, $\left.\left(M_{s}^{\otimes 2}\right)_{2:}\right)$ means that in the tensor of order $2, M_{s}^{\otimes 2}=\left(M_{s}^{\otimes 2}\right)_{i j=1,2}$, we fix $i=1$ (respectively, $i=2$ ) and let $j$ vary. Using (4.9) and (4.12) with $m=2$ we decompose

$$
M_{s}^{\otimes 2}=\left[\begin{array}{c}
\mathrm{d} \otimes \operatorname{Id}\left(M_{s}^{\circ}\right)_{1:}+\left(M_{s}^{\perp}\right)_{1:} \\
\mathrm{d} \otimes \operatorname{Id}\left(M_{s}^{\circ}\right)_{2:}+\left(M_{s}^{\perp}\right)_{2:}
\end{array}\right] \in\left[\begin{array}{c}
U_{k}^{2} \\
U_{k-1}^{2}
\end{array}\right],
$$

where

$$
\begin{aligned}
& \left(M_{s}^{\perp}\right)_{1:}=\pi^{\perp} \otimes \operatorname{Id}\left(M_{s}^{\otimes 2}\right)_{1:} \in \mathfrak{B}_{k}^{2, \perp}, \\
& \left(M_{s}^{\perp}\right)_{2:}=\pi^{\perp} \otimes \operatorname{Id}\left(M_{s}^{\otimes 2}\right)_{2:} \in \mathfrak{B}_{k-1}^{2, \perp}, \\
& \left(M_{s}^{\circ}\right)_{1:}=\pi^{\circ} \otimes \operatorname{Id}\left(M_{s}^{\otimes 2}\right)_{1:} \in \mathfrak{B}_{k-1}^{2, \perp}, \\
& \left(M_{s}^{\circ}\right)_{2:}=\pi^{\circ} \otimes \operatorname{Id}\left(M_{s}^{\otimes 2}\right)_{2:} \in \mathfrak{B}_{k-2}^{2, \perp} .
\end{aligned}
$$

We choose $M_{t}^{\otimes 2}=P \otimes P M_{s}^{\otimes 2}$, where $P$ is defined in (2.22), so that

$$
\begin{aligned}
\left\langle T \otimes T M_{s}^{\otimes 2}, M_{t}^{\otimes 2}\right\rangle & =\left\langle T \otimes T M_{s}^{\otimes 2}, P \otimes P M_{s}^{\otimes 2}\right\rangle \\
& =\sum_{i, j=1}^{2}\left\langle T_{i j} \otimes T\left(M_{s}^{\otimes 2}\right)_{j:},\left(P \otimes P M_{s}^{\otimes 2}\right)_{i:}\right\rangle .
\end{aligned}
$$

Let $\left\langle T_{i j} \otimes T\left(M_{s}^{\otimes 2}\right)_{j:},\left(P \otimes P M_{s}^{\otimes 2}\right)_{i:}\right\rangle=\mathcal{I}_{i j}$. We will bound each term $\mathcal{I}_{i j}$ for $i, j=1,2$.

Using (4.2) we make explicit the term $\left(P \otimes P M_{s}^{\otimes 2}\right)_{i:}$ :

$$
\left(P \otimes P M_{s}^{\otimes 2}\right)_{i:}=P_{i 1} \otimes P\left(M_{s}^{\otimes 2}\right)_{1:}+P_{i 2} \otimes P\left(M_{s}^{\otimes 2}\right)_{2:} .
$$


Let us start from the case $i=j=1$ :

$$
\mathcal{I}_{11}=\left\langle A \otimes T\left(M_{s}^{\otimes 2}\right)_{1:},\left(\pi^{\perp} \otimes P\left(M_{s}^{\otimes 2}\right)_{1:}+\mathrm{d} \pi^{\perp} \otimes P\left(M_{s}^{\otimes 2}\right)_{2:}\right)\right\rangle .
$$

Since $\mathrm{d} \circ \mathrm{d}=0$, then $\left.\left\langle A \otimes T\left(M_{s}^{\otimes 2}\right)_{1:}, \mathrm{d} \pi^{\perp} \otimes P\left(M_{s}^{\otimes 2}\right)_{2:}\right)\right\rangle=0$ and $A \otimes T\left(\mathrm{~d} \otimes \operatorname{Id} M_{s}^{\circ}\right)_{1:} \equiv 0$. Hence,

$$
\begin{aligned}
\mathcal{I}_{11} & =\left\langle A \otimes T\left(M_{s}^{\perp}\right)_{1:}, \mathrm{Id} \otimes P\left(M_{s}^{\perp}\right)_{1:}\right\rangle \\
& =\left\langle\mathrm{d} \otimes T\left(M_{s}^{\perp}\right)_{1:}, \mathrm{d} \otimes P\left(M_{s}^{\perp}\right)_{1:}\right\rangle \\
& \geqslant C_{1}\left\|\mathrm{~d} \otimes \operatorname{Id}\left(M_{s}^{\perp}\right)_{1:}\right\|_{L^{2} \Lambda^{k+1} \otimes V_{k}}^{2} .
\end{aligned}
$$

The last step follows from Theorem 2.6. If $i=1$ and $j=2$, we find

$$
\mathcal{I}_{12}=\left\langle B^{*} \otimes T\left(M_{s}^{\otimes 2}\right)_{2:}, \pi^{\perp} \otimes P\left(M_{s}^{\otimes 2}\right)_{1:}+\mathrm{d} \pi^{\perp} \otimes P\left(M_{s}^{\otimes 2}\right)_{2:}\right\rangle .
$$

Since $\pi^{\perp} \otimes P\left(M_{s}^{\otimes 2}\right)_{1:} \in \mathfrak{B}_{k}^{2, \perp}$, then $\left\langle B^{*} \otimes T\left(M_{s}^{\otimes 2}\right)_{2:}, \pi^{\perp} \otimes P\left(M_{s}^{\otimes 2}\right)_{1:}\right\rangle=0$. Hence,

$$
\begin{aligned}
\mathcal{I}_{12} & =\left\langle B^{*} \otimes T\left(M_{s}^{\perp}\right)_{2:}, \mathrm{d} \otimes P\left(M_{s}^{\perp}\right)_{2:}\right\rangle \\
& =\left\langle\mathrm{d} \otimes T\left(M_{s}^{\perp}\right)_{2:}, \mathrm{d} \otimes P\left(M_{s}^{\perp}\right)_{2:}\right\rangle \\
& \geqslant C_{1}\left\|\mathrm{~d} \otimes \operatorname{Id}\left(M_{s}^{\perp}\right)_{2:}\right\|_{L^{2} \Lambda^{k} \otimes V_{k}}^{2} .
\end{aligned}
$$

If $i=2$ and $j=1$, we find

$$
\mathcal{I}_{21}=\left\langle B \otimes T\left(M_{s}^{\otimes 2}\right)_{1:}, \gamma \pi^{\circ} \otimes P\left(M_{s}^{\otimes 2}\right)_{1:}-\mathrm{d} \pi^{\circ} \otimes P\left(M_{s}^{\otimes 2}\right)_{2:}\right\rangle .
$$

Since $\left\langle B \otimes T\left(M_{s}^{\otimes 2}\right)_{1:}, \mathrm{d} \pi^{\circ} \otimes P\left(M_{s}^{\otimes 2}\right)_{2:}\right\rangle=0$ and $\left\langle B \otimes T\left(M_{s}^{\perp}\right)_{1:}, \operatorname{Id} \otimes P\left(M_{s}^{\circ}\right)_{1:}\right\rangle=0$, we have

$$
\begin{aligned}
\mathcal{I}_{21} & =\gamma\left\langle B \otimes T\left(\mathrm{~d} \otimes \operatorname{Id}\left(M_{s}^{\circ}\right)_{1:}\right), \operatorname{Id} \otimes P\left(M_{s}^{\circ}\right)_{1:}\right\rangle \\
& =\gamma\left\langle\mathrm{d} \otimes T\left(M_{s}^{\circ}\right)_{1:}, \mathrm{d} \otimes P\left(M_{s}^{\circ}\right)_{1:}\right\rangle \\
& \geqslant \gamma C_{1}\left\|\mathrm{~d} \otimes \operatorname{Id}\left(M_{s}^{\circ}\right)_{1:}\right\|_{L^{2} \Lambda^{k-1} \otimes V_{k}}^{2} .
\end{aligned}
$$

If $i=j=2$, then

$$
\begin{aligned}
\mathcal{I}_{22}= & -\alpha\left\langle\operatorname{Id} \otimes T\left(M_{s}^{\otimes 2}\right)_{2:}, \gamma \pi^{\circ} \otimes P\left(M_{s}^{\otimes 2}\right)_{1:}-\mathrm{d} \pi^{\circ} \otimes P\left(M_{s}^{\otimes 2}\right)_{2:}\right\rangle \\
= & \alpha\left\langle\operatorname{Id} \otimes T\left(M_{s}^{\otimes 2}\right)_{2:}, \mathrm{d} \pi^{\circ} \otimes P\left(M_{s}^{\otimes 2}\right)_{2:}\right\rangle \\
& -\alpha\left\langle\operatorname{Id} \otimes T\left(M_{s}^{\otimes 2}\right)_{2:}, \gamma \pi^{\circ} \otimes P\left(M_{s}^{\otimes 2}\right)_{1:}\right\rangle .
\end{aligned}
$$

Since $\left\langle\mathrm{Id} \otimes T\left(M_{s}^{\perp}\right)_{2:}, \mathrm{d} \pi^{\circ} \otimes P\left(M_{s}^{\otimes 2}\right)_{2:}\right\rangle=0$, we find

$$
\begin{aligned}
(4.18) & =\alpha\left\langle\mathrm{d} \otimes T\left(M_{s}^{\circ}\right)_{2:}, \mathrm{d} \otimes P\left(M_{s}^{\circ}\right)_{2:}\right\rangle \\
& \geqslant \alpha C_{1}\left\|\mathrm{~d} \otimes \operatorname{Id}\left(M_{s}^{\circ}\right)_{2:}\right\|_{L^{2} \Lambda^{k-1} \otimes V_{k}}^{2}
\end{aligned}
$$


Moreover, since $\left\langle\operatorname{Id} \otimes T\left(\mathrm{~d} \pi^{\circ} \otimes \operatorname{Id}\left(M_{s}^{\otimes 2}\right)_{2:}\right), \pi^{\circ} \otimes P\left(M_{s}^{\otimes 2}\right)_{1:}\right\rangle=0$, we find

$$
\begin{aligned}
(4.19)= & -\alpha \gamma\left\langle\operatorname{Id} \otimes T\left(M_{s}^{\perp}\right)_{2:}, \operatorname{Id} \otimes P\left(M_{s}^{\circ}\right)_{1:}\right\rangle \\
\geqslant & -\frac{\alpha}{2} \gamma^{1 / 2}\left(\left\|\operatorname{Id} \otimes T\left(M_{s}^{\perp}\right)_{2:}\right\|_{L^{2} \Lambda^{k-1} \otimes V_{k}^{\prime}}^{2}+\gamma\left\|\operatorname{Id} \otimes P\left(M_{s}^{\circ}\right)_{1:}\right\|_{L^{2} \Lambda^{k} \otimes V_{k}}^{2}\right) \\
\geqslant & -\frac{\alpha}{2} \gamma^{1 / 2}\left(C_{P, 1}^{2}\|T\|_{\mathcal{L}\left(V_{k}, V_{k}^{\prime}\right)}^{2}\left\|\mathrm{~d} \otimes \operatorname{Id}\left(M_{s}^{\perp}\right)_{2:}\right\|_{L^{2} \Lambda^{k} \otimes V_{k}}^{2}\right. \\
& \left.+\gamma C_{P, 1}^{2}\|P\|_{\mathcal{L}\left(V_{k}, V_{k}\right)}^{2}\left\|\mathrm{~d} \otimes \operatorname{Id}\left(M_{s}^{\circ}\right)_{1:}\right\|_{L^{2} \Lambda^{k} \otimes V_{k}}^{2}\right)
\end{aligned}
$$

where we used Proposition 4.1 and Lemma 4.7. Using the lower bounds on $\mathcal{I}_{11}, \mathcal{I}_{12}, \mathcal{I}_{21}$ and $\mathcal{I}_{22}$, we can now conclude that

$$
\begin{aligned}
(4.17) \geqslant & C_{1}\left\|\mathrm{~d} \otimes \operatorname{Id}\left(M_{s}^{\perp}\right)_{1:}\right\|_{L^{2} \Lambda^{k+1} \otimes V_{k}}^{2} \\
& +\left(C_{1}-\frac{\alpha}{2} \gamma^{1 / 2} C_{P, 1}^{2}\|T\|_{\mathcal{L}\left(V_{k}, V_{k}^{\prime}\right)}^{2}\right)\left\|\mathrm{d} \otimes \operatorname{Id}\left(M_{s}^{\perp}\right)_{2:}\right\|_{L^{2} \Lambda^{k} \otimes V_{k}}^{2} \\
& +\gamma\left(C_{1}-\frac{\alpha}{2} \gamma^{1 / 2} C_{P, 1}^{2}\|P\|_{\mathcal{L}\left(V_{k}, V_{k}\right)}^{2}\right)\left\|\mathrm{d} \otimes \operatorname{Id}\left(M_{s}^{\circ}\right)_{1:}\right\|_{L^{2} \Lambda^{k} \otimes V_{k}}^{2} \\
& +\alpha C_{1}\left\|\mathrm{~d} \otimes \operatorname{Id}\left(M_{s}^{\circ}\right)_{2:}\right\|_{L^{2} \Lambda^{k-1} \otimes V_{k}}^{2}
\end{aligned}
$$

Hence, if we choose $\gamma$ sufficiently small, condition (4.7) is satisfied for $m=2$. Now suppose that the problem for the $(m-1)$ th moment is well posed and in particular that the inf-sup condition is verified with the test function $M_{t}^{\otimes(m-1)}=P^{\otimes(m-1)} M_{s}^{\otimes(m-1)}$ :

$$
\left\langle T^{\otimes(m-1)} M_{s}^{\otimes(m-1)}, P^{\otimes(m-1)} M_{s}^{\otimes(m-1)}\right\rangle \geqslant C_{m-1}\left\|M_{s}^{\otimes(m-1)}\right\|_{V_{k}^{\otimes(m-1)}}^{2},
$$

where $C_{m-1}=C_{m-1}\left(C_{P, 1}, \alpha,\|T\|,\|P\|\right)>0$. We want to prove (4.7). As before, we fix $M_{s}^{\otimes m}=\left[\begin{array}{l}\left(M_{s}^{\otimes m}\right)_{1:} \\ \left(M_{s}^{\otimes m}\right)_{2:}\end{array}\right]$ where $\left(M_{s}^{\otimes m}\right)_{1:}$ (respectively, $\left(M_{s}^{\otimes m}\right)_{2:}$ ) means that in the tensor of order $m, M_{s}^{\otimes m}=\left(M_{s}^{\otimes m}\right)_{i_{1} \ldots i_{m}=1,2}$, we fix $i_{1}=1$ (respectively, $i_{1}=2$ ) and let $i_{2}, \ldots, i_{m}$ vary. Using (4.9) and (4.12) we decompose

$$
M_{s}^{\otimes m}=\left[\begin{array}{c}
\left(M_{s}^{\perp}\right)_{1:}+\mathrm{d} \otimes \mathrm{Id}^{\otimes(m-1)}\left(M_{s}^{\circ}\right)_{1:} \\
\left(M_{s}^{\perp}\right)_{2:}+\mathrm{d} \otimes \mathrm{Id}^{\otimes(m-1)}\left(M_{s}^{\circ}\right)_{2:}
\end{array}\right] \in\left[\begin{array}{c}
U_{k}^{m} \\
U_{k-1}^{m}
\end{array}\right],
$$

where now

$$
\begin{aligned}
& \left(M_{s}^{\perp}\right)_{1:}=\pi^{\perp} \otimes \mathrm{Id}^{\otimes(m-1)}\left(M_{s}^{\otimes m}\right)_{1:} \in \mathfrak{B}_{k}^{m, \perp}, \\
& \left(M_{s}^{\perp}\right)_{2:}=\pi^{\perp} \otimes \mathrm{Id}^{\otimes(m-1)}\left(M_{s}^{\otimes m}\right)_{2:} \in \mathfrak{B}_{k-1}^{m, \perp}, \\
& \left(M_{s}^{\circ}\right)_{1:}=\pi^{\circ} \otimes \operatorname{Id}^{\otimes(m-1)}\left(M_{s}^{\otimes m}\right)_{1:} \in \mathfrak{B}_{k-1}^{m, \perp}, \\
& \left(M_{s}^{\circ}\right)_{2:}=\pi^{\circ} \otimes \operatorname{Id}^{\otimes(m-1)}\left(M_{s}^{\otimes m}\right)_{2:} \in \mathfrak{B}_{k-2}^{m, \perp} .
\end{aligned}
$$


We choose $M_{t}^{\otimes m}=P^{\otimes m} M_{s}^{\otimes m}$, so that

$$
\begin{aligned}
\left\langle T^{\otimes m} M_{s}^{\otimes m}, M_{t}^{\otimes m}\right\rangle & =\left\langle T^{\otimes m} M_{s}^{\otimes m}, P^{\otimes m} M_{s}^{\otimes m}\right\rangle \\
& =\sum_{i, j=1}^{2}\left\langle T_{i, j} \otimes T^{\otimes(m-1)}\left(M_{s}^{\otimes m}\right)_{j:},\left(P^{\otimes m} M_{s}^{\otimes m}\right)_{i:}\right\rangle .
\end{aligned}
$$

Let $\mathcal{J}_{i j}=\left\langle T_{i, j} \otimes T^{m-1}\left(M_{s}^{\otimes m}\right)_{j:},\left(P^{\otimes m} M_{s}^{\otimes m}\right)_{i:}\right\rangle$. We follow the same reasoning as before, and we apply (4.20). If $i=j=1$, then

$$
\begin{aligned}
\mathcal{J}_{11} & =\left\langle A \otimes T^{\otimes(m-1)}\left(M_{s}^{\otimes m}\right)_{1:},\left(P \otimes P^{\otimes(m-1)} M_{s}^{\otimes m}\right)_{1:}\right\rangle \\
& \geqslant C_{m-1}\left\|\mathrm{~d} \otimes \mathrm{Id}^{\otimes(m-1)}\left(M_{s}^{\perp}\right)_{1:}\right\|_{L^{2} \Lambda^{k+1} \otimes V_{k}^{\otimes(m-1)} .}^{2}
\end{aligned}
$$

If $i=1$ and $j=2$, then

$$
\begin{aligned}
\mathcal{J}_{12} & =\left\langle B^{*} \otimes T^{\otimes(m-1)}\left(M_{s}^{\otimes m}\right)_{2:},\left(P \otimes P^{\otimes(m-1)} M_{s}^{\otimes m}\right)_{1:}\right\rangle \\
& \geqslant C_{m-1}\left\|\mathrm{~d} \otimes \operatorname{Id}^{\otimes(m-1)}\left(M_{s}^{\perp}\right)_{2:}\right\|_{L^{2} \Lambda^{k} \otimes V_{k}^{\otimes(m-1)} .}^{2}
\end{aligned}
$$

If $i=2$ and $j=1$, then

$$
\begin{aligned}
\mathcal{J}_{21} & =\left\langle B \otimes T^{\otimes(m-1)}\left(M_{s}^{\otimes m}\right)_{1:},\left(P \otimes P^{\otimes(m-1)} M_{s}^{\otimes m}\right)_{2:}\right\rangle \\
& \geqslant \gamma C_{m-1}\left\|\mathrm{~d} \otimes \mathrm{Id}^{\otimes(m-1)}\left(M_{s}^{\circ}\right)_{1:}\right\|_{L^{2} \Lambda^{k} \otimes V_{k}^{\otimes(m-1)}}^{2} .
\end{aligned}
$$

If $i=j=2$, then

$$
\begin{aligned}
\mathcal{J}_{22}= & -\alpha\left\langle\mathrm{Id} \otimes T^{\otimes(m-1)}\left(M_{s}^{\otimes m}\right)_{2:},\left(P \otimes P^{\otimes(m-1)} M_{s}^{\otimes m}\right)_{2:}\right\rangle \\
\geqslant & \alpha C_{m-1}\left\|\mathrm{~d} \otimes \operatorname{Id}^{\otimes(m-1)}\left(M_{s}^{\circ}\right)_{2:}\right\|_{L^{2} \Lambda^{k-1} \otimes V_{k}^{\otimes(m-1)}}^{2} \\
& -\frac{\alpha}{2} \gamma^{1 / 2}\left(C_{P, 1}^{2}\|T\|_{\mathcal{L}\left(V_{k}, V_{k}^{\prime}\right)}^{2(m-1)}\left\|\mathrm{d} \otimes \mathrm{Id}^{\otimes(m-1)}\left(M_{s}^{\perp}\right)_{2:}\right\|_{L^{2} \Lambda^{k} \otimes V_{k}^{\otimes(m-1)}}^{2}\right. \\
& +\gamma C_{P, 1}^{2}\|P\|_{\mathcal{L}\left(V_{k}, V_{k}\right)}^{2(m-1)}\left\|\mathrm{d} \otimes \mathrm{Id}^{\otimes(m-1)}\left(M_{s}^{\circ}\right)_{1:}\right\|_{\left.L^{2} \Lambda^{k} \otimes V_{k}^{\otimes(m-1)}\right) .}^{2}
\end{aligned}
$$

Hence, if we choose $\gamma$ sufficiently small, condition (4.7) is satisfied. Relation (4.8) follows from the orthogonal decomposition (4.12) and the tensorial Poincaré inequality in Lemma 4.7.

REMARK 4.8 We underline that the operator $P$ is not the classical one presented in Arnold et al. (2006) to prove the well-posedness of the deterministic Hodge-Laplace problem. Indeed it is such that the inf-sup condition for $\left\langle T^{\otimes m} \cdot, \cdot\right\rangle: V_{k}^{\otimes m} \times V_{k}^{\otimes m} \rightarrow \mathbb{R}$ (for every finite $m \geqslant 1$ ) is satisfied. With the classical operator, the inf-sup condition for $m \geqslant 2$ is not automatically satisfied.

\section{Some three-dimensional problems important in applications}

In Section 2.2.1, we reinterpreted the deterministic Hodge-Laplace problem in $n=3$ dimensions in terms of PDEs. Here, we translate in terms of PDEs the stochastic Hodge-Laplace problem. In particular, we focus on the two problems obtained for $k=1$ and $k=3$ : the stochastic magnetostatic/electrostatic 
equations and the stochastic Darcy equations, and we explicitly write the systems solved by the mean and the 2-points correlation of the unique stochastic solution of the stochastic problem.

\subsection{The stochastic magnetostatic/electrostatic equations}

Take $k=1$ and $\alpha=0$. Let $f_{1} \in L^{m}\left(\Omega ; L^{2} \Lambda^{1}(D)\right), f_{2} \in L^{m}\left(\Omega ; L^{2} \Lambda^{0}(D)\right)$ be stochastic functions with $m \geqslant 1$ integer, representing an uncertain current and an uncertain charge, respectively. The stochastic magnetostatic/electrostatic problem is the stochastic counterpart of problem (2.30). Owing to Theorem 3.1, the stochastic magnetostatic/electrostatic problem admits a unique stochastic solution that depends continuously on the data. If $m \geqslant 1$, the first statistical moment $\mathcal{M}^{1}\left[\begin{array}{l}u \\ p\end{array}\right]=\mathbb{E}\left[\begin{array}{l}u \\ p\end{array}\right]$ is well defined, and is the unique solution of the following problem: find $E_{s}=\left[\begin{array}{l}E_{s, 1} \\ E_{s, 2}\end{array}\right] \in V_{1}$ such that

$$
\left\{\begin{array}{l}
\left(\operatorname{curl} E_{s, 1}, \operatorname{curl} v\right)+\left(\nabla E_{s, 2}, v\right)=\left(\mathbb{E}\left[f_{1}\right], v\right), \quad \forall\left[\begin{array}{l}
v \\
q
\end{array}\right] \in V_{1},
\end{array}\right.
$$

where the parentheses in (5.1) mean the $L^{2}$ inner product. In the case $m \geqslant 2$, the second statistical moment $\mathcal{M}^{2}\left[\begin{array}{l}u \\ p\end{array}\right]$ is well defined and is the unique solution of the following (see (4.5) with $m=2$ ): find

$$
M_{s}^{\otimes 2} \in V_{1} \otimes V_{1}=\left[\begin{array}{cc}
H_{\Gamma_{\mathrm{D}}}(\operatorname{curl}, D) \otimes H_{\Gamma_{\mathrm{D}}}(\operatorname{curl}, D) & H_{\Gamma_{\mathrm{D}}}(\operatorname{curl}, D) \otimes H_{\Gamma_{\mathrm{D}}}^{1}(D) \\
H_{\Gamma_{\mathrm{D}}}^{1}(D) \otimes H_{\Gamma_{\mathrm{D}}}(\operatorname{curl}, D) & H_{\Gamma_{\mathrm{D}}}^{1}(D) \otimes H_{\Gamma_{\mathrm{D}}}^{1}(D)
\end{array}\right]
$$

such that

$$
\left\{\begin{aligned}
&\left(\operatorname{curl} \otimes \operatorname{curl}\left(M_{s}^{\otimes 2}\right)_{11}, \operatorname{curl} \otimes \operatorname{curl}\left(M_{t}^{\otimes 2}\right)_{11}\right)+\left(\operatorname{curl} \otimes \nabla\left(M_{s}^{\otimes 2}\right)_{12}, \operatorname{curl} \otimes \operatorname{Id}\left(M_{t}^{\otimes 2}\right)_{11}\right) \\
&+\left(\nabla \otimes \operatorname{curl}\left(M_{s}^{\otimes 2}\right)_{21}, \operatorname{Id} \otimes \operatorname{curl}\left(M_{t}^{\otimes 2}\right)_{11}\right)+\left(\nabla \otimes \nabla\left(M_{s}^{\otimes 2}\right)_{22},\left(M_{t}^{\otimes 2}\right)_{11}\right) \\
&=\left(\mathcal{M}^{2}\left[f_{1}\right],\left(M_{t}^{\otimes 2}\right)_{11}\right), \\
&-\left(\operatorname{curl} \otimes \operatorname{Id}\left(M_{s}^{\otimes 2}\right)_{11}, \operatorname{curl} \otimes \nabla\left(M_{t}^{\otimes 2}\right)_{12}\right)-\left(\nabla \otimes \operatorname{Id}\left(M_{s}^{\otimes 2}\right)_{12}, \operatorname{Id} \otimes \nabla\left(M_{t}^{\otimes 2}\right)_{12}\right) \\
&=\left(\mathbb{E}\left[f_{1} f_{2}\right],\left(M_{t}^{\otimes 2}\right)_{12}\right), \\
&-\left(\operatorname{Id} \otimes \operatorname{curl}\left(M_{s}^{\otimes 2}\right)_{12}, \nabla \otimes \operatorname{curl}\left(M_{t}^{\otimes 2}\right)_{21}\right)-\left(\operatorname{Id} \otimes \nabla\left(M_{s}^{\otimes 2}\right)_{21}, \nabla \otimes \operatorname{Id}\left(M_{t}^{\otimes 2}\right)_{21}\right) \\
&=\left(\mathbb{E}\left[f_{2} f_{1}\right],\left(M_{t}^{\otimes 2}\right)_{21}\right), \\
&\left(\left(M_{s}^{\otimes 2}\right)_{11}, \nabla \otimes \nabla\left(M_{t}^{\otimes 2}\right)_{22}\right)=\left(\mathcal{M}^{2}\left[f_{2}\right],\left(M_{t}^{\otimes 2}\right)_{22}\right),
\end{aligned}\right.
$$

for all $M_{t}^{\otimes 2} \in V_{1} \otimes V_{1}$, where the parentheses in (5.2) denote the $L^{2}$-inner product either between scalar or vector functions.

\subsection{The stochastic Darcy problem}

Let $k=3, f_{2} \equiv 0$ and let $f_{1} \in L^{m}\left(\Omega ; L^{2} \Lambda^{3}(D)\right)$, with $m \geqslant 1$ integer, represent an uncertain source in porous media flow. The stochastic Darcy problem is the stochastic counterpart of problem (2.33). Thanks to Theorem 3.1, the stochastic Darcy problem admits a unique stochastic solution that depends continuously on the data. If $m \geqslant 1$, the first statistical moment $\mathcal{M}^{1}\left[\begin{array}{l}u \\ p\end{array}\right]=\mathbb{E}\left[\begin{array}{l}u \\ p\end{array}\right]$ is well defined and is 
the unique solution of the following: find $E_{s}=\left[\begin{array}{c}E_{s, 1} \\ E_{s, 2}\end{array}\right] \in V_{3}$ such that

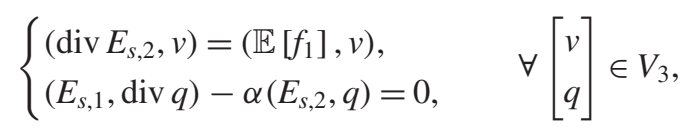

where the parentheses in (5.3) mean the $L^{2}$ inner product. In the case $m \geqslant 2$, the second statistical moment $\mathcal{M}^{2}\left[\begin{array}{l}u \\ p\end{array}\right]$ is well defined and is the unique solution of the following (see (4.5) with $m=2$ ): find

$$
M_{s}^{\otimes 2} \in V_{3} \otimes V_{3}=\left[\begin{array}{cc}
L^{2}(D) \otimes L^{2}(D) & L^{2}(D) \otimes H_{\Gamma_{\mathrm{D}}}(\operatorname{div}, D) \\
H_{\Gamma_{\mathrm{D}}}(\operatorname{div}, D) \otimes L^{2}(D) & H_{\Gamma_{\mathrm{D}}}(\operatorname{div} ; D) \otimes H_{\Gamma_{\mathrm{D}}}(\operatorname{div} ; D)
\end{array}\right]
$$

such that

$$
\left\{\begin{array}{l}
\left(\operatorname{div} \otimes \operatorname{div}\left(M_{s}^{\otimes 2}\right)_{22},\left(M_{t}\right)_{11}\right)=\left(\mathcal{M}^{2}\left[f_{1}\right],\left(M_{t}\right)_{11}\right), \\
\left(\operatorname{div} \otimes \operatorname{Id}\left(M_{s}^{\otimes 2}\right)_{21}, \operatorname{Id} \otimes \operatorname{div}\left(M_{t}^{\otimes 2}\right)_{12}\right)-\alpha\left(\operatorname{div} \otimes \operatorname{Id}\left(M_{s}^{\otimes 2}\right)_{22},\left(M_{t}^{\otimes 2}\right)_{12}\right)=0, \\
\left(\operatorname{Id} \otimes \operatorname{div}\left(M_{s}^{\otimes 2}\right)_{12}, \operatorname{div} \otimes \operatorname{Id}\left(M_{t}^{\otimes 2}\right)_{21}\right)-\alpha\left(\operatorname{Id} \otimes \operatorname{div}\left(M_{s}^{\otimes 2}\right)_{22},\left(M_{t}^{\otimes 2}\right)_{21}\right)=0, \\
\left(\left(M_{s}^{\otimes 2}\right)_{11}, \operatorname{div} \otimes \operatorname{div}\left(M_{t}^{\otimes 2}\right)_{22}\right)-\alpha\left(\left(M_{s}^{\otimes 2}\right)_{12}, \operatorname{div} \otimes \operatorname{Id}\left(M_{t}^{\otimes 2}\right)_{22}\right) \\
\quad-\alpha\left(\left(M_{s}^{\otimes 2}\right)_{21}, \operatorname{Id} \otimes \operatorname{div}\left(M_{t}^{\otimes 2}\right)_{22}\right)+\alpha^{2}\left(\left(M_{s}^{\otimes 2}\right)_{22},\left(M_{t}^{\otimes 2}\right)_{22}\right)=0,
\end{array}\right.
$$

for all $M_{t}^{\otimes 2} \in V_{3} \otimes V_{3}$, where the parentheses in (5.4) denote the $L^{2}$-inner product either between scalar or vector functions.

\section{Finite element discretization of the moment equations}

In this section, we aim to derive a stable discretization for the moment equations, that is, the deterministic problems solved by the statistics of the unique stochastic solution $\left[\begin{array}{l}u \\ p\end{array}\right]$. First, we recall the main concepts concerning the finite element differential forms and the existence of a stable finite element discretization for the mean problem. Then, we construct both a full and a sparse tensor product finite element discretization for the $m$ th problem, with $m \geqslant 2$ integer, we prove their stability and provide optimal order-of-convergence estimates.

\subsection{Finite element differential forms and the discrete mean problem}

Following Arnold et al. (2006), throughout this section we assume that the domain $D \subset \mathbb{R}^{n}$ is a polyhedral domain in $\mathbb{R}^{n}$ which is partitioned into a finite set of $n$-simplices. These simplices are such that their union is the closure of $D$ and the intersection of any two of them, if nonempty, is a common subsimplex. We denote the partition with $\mathcal{T}_{h}$ and the discretization parameter with $h$. To discretize the moment equations we use the finite element differential forms

$$
\mathcal{P}_{r}^{-} \Lambda^{k}\left(\mathcal{T}_{h}\right)=\left\{v \in H \Lambda^{k}(D)|v|_{T} \in \mathcal{P}_{r}^{-} \Lambda^{k}(T) \forall T \in \mathcal{T}_{h}\right\},
$$

where the space $\mathcal{P}_{r}^{-} \Lambda^{k}(T)$ and the de Rham subcomplex

$$
0 \rightarrow \mathcal{P}_{r}^{-} \Lambda^{0}\left(\mathcal{T}_{h}\right) \stackrel{\mathrm{d}}{\rightarrow} \cdots \stackrel{\mathrm{d}}{\rightarrow} \mathcal{P}_{r}^{-} \Lambda^{n}\left(\mathcal{T}_{h}\right) \rightarrow 0
$$


TABLE 2 Proxy-field correspondences between finite element differential forms $\mathcal{P}_{r}^{-} \Lambda^{k}\left(\mathcal{T}_{h}\right)$ and the classical finite element spaces for $n=3$

\begin{tabular}{lll}
\hline$k=0$ & $\mathcal{P}_{r}^{-} \Lambda^{0}\left(\mathcal{T}_{h}\right)$ & Lagrangian elements of degree $\leqslant r$ \\
$k=1$ & $\mathcal{P}_{r}^{-} \Lambda^{1}\left(\mathcal{T}_{h}\right)$ & Nédélec first kind $H$ (curl) elements of order $r-1$ \\
$k=2$ & $\mathcal{P}_{r}^{-} \Lambda^{2}\left(\mathcal{T}_{h}\right)$ & Nédélec first kind $H($ div) elements of order $r-1$ \\
$k=3$ & $\mathcal{P}_{r}^{-} \Lambda^{3}\left(\mathcal{T}_{h}\right)$ & Discontinuous elements of degree $\leqslant r-1$ \\
\hline
\end{tabular}

are treated in Hiptmair (2002) and Arnold et al. (2006). Since we are particularly interested in the $n=3$ case, we recall in Table 2 the correspondences between the finite element differential forms (6.1) and the classical finite element spaces of scalar and vector functions. The spaces $\mathcal{P}_{r}^{-} \Lambda^{k}\left(\mathcal{T}_{h}\right)$ are not the only choice. Indeed, in Hiptmair (2002), Arnold et al. (2006, 2010) and Christiansen et al. (2011), the authors present other finite element differential forms to discretize the deterministic Hodge Laplacian.

In Arnold et al. (2010), the authors propose the construction of a projector

$$
\Pi_{k, h}: H \Lambda^{k}(D) \rightarrow \mathcal{P}_{r}^{-} \Lambda^{k}\left(\mathcal{T}_{h}\right)
$$

which is a cochain map, that is, it commutes with the exterior derivative, and such that the following approximation property holds:

$$
\left\|v-\Pi_{k, h} v\right\|_{L^{2} \Lambda^{k}} \leqslant C h^{s}\|v\|_{H^{s} \Lambda^{k}} \quad \forall v \in H^{s} \Lambda^{k}(D), 0 \leqslant s \leqslant r
$$

where $C$ is independent of $h$. Note that the inequality (6.2) for $s=0$ implies the stability of the projector in $L^{2}$. Moreover, from (6.2) it follows the boundedness of the projector $\Pi_{k, h}$ in the $H \Lambda^{k}(D)$ norm. Since we are dealing with Dirichlet boundary conditions on $\Gamma_{\mathrm{D}}$, we need the existence of cochain projectors which also respect the boundary conditions. To this aim, we make the following assumption.

Assumption 6.1 There exists a bounded cochain projector, that, by abuse of notation, we denote still by $\Pi_{k, h}$ (and, when no ambiguity arises, by $\Pi_{h}$ ),

$$
\Pi_{k, h}: H_{\Gamma_{\mathrm{D}}} \Lambda^{k}(D) \rightarrow \mathcal{P}_{r, \Gamma_{\mathrm{D}}}^{-} \Lambda^{k}\left(\mathcal{T}_{h}\right):=\mathcal{P}_{r}^{-} \Lambda^{k}\left(\mathcal{T}_{h}\right) \cap H_{\Gamma_{\mathrm{D}}} \Lambda^{k}(D)
$$

such that (6.2) is satisfied for every $v \in H^{s} \Lambda^{k}(D) \cap H_{\Gamma_{\mathrm{D}}} \Lambda^{k}(D), 0 \leqslant s \leqslant r$.

Assumption 6.1 is satisfied in the two- and three-dimensional cases; see Schöberl (2008). The $n$-dimensional case is still a topic of current research, whereas if natural boundary conditions are imposed on $\partial D$, the existence of such an operator is proved in Arnold et al. (2006), and if essential boundary conditions are imposed on $\partial D$, the existence of such an operator is proved in Christiansen $\&$ Winther (2008).

The problem solved by the mean of the unique stochastic solution of the stochastic Hodge Laplacian turns out to be the deterministic Hodge Laplacian. In Arnold et al. (2006), the authors study the finite element formulation of the deterministic Hodge Laplacian with natural boundary conditions on $\partial D$ $\left(\Gamma_{\mathrm{D}}=\emptyset\right)$. In Arnold et al. (2010), all the results obtained in Arnold et al. (2006) for $\Gamma_{\mathrm{D}}=\emptyset$ are extended to include the case of essential boundary conditions on $\partial D\left(\Gamma_{\mathrm{N}}=\emptyset\right)$. Under Assumption 6.1, all the results in Arnold et al. $(2006,2010)$ apply to the general case $\Gamma_{\mathrm{D}}, \Gamma_{\mathrm{N}} \neq \emptyset$. In particular, the finite element formulation of the mean problem is well posed. Moreover, using a quasi-optimal error estimate 
and the interpolation property (6.2), we get the following order-of-convergence estimate:

$$
\left\|\mathbb{E}\left[\begin{array}{l}
u \\
p
\end{array}\right]-E_{s, h}\right\|_{V_{k}}=\mathcal{O}\left(h^{r}\right)
$$

$\mathbb{E}\left[\begin{array}{l}u \\ p\end{array}\right]$ and $E_{s, h}$ being the unique solutions of the continuous and discrete mean problems, respectively.

\subsection{Discrete mth moment problem: FTP approximation}

The FTP finite element (FTP-FE) formulation of problem (4.5) is as follows.

\section{m-Points Correlation Problem (FTP-FE)}

$$
\begin{gathered}
\text { Given } m \geqslant 2 \text { integer and }\left[\begin{array}{l}
F_{1} \\
F_{2}
\end{array}\right] \in L^{m}\left(\Omega ; V_{k}^{\prime}\right) \text {, find } M_{s, h}^{\otimes m} \in V_{k, h}^{\otimes m} \text { such that } \\
T^{\otimes m} M_{s, h}^{\otimes m}=\mathcal{M}^{m}\left[\begin{array}{l}
F_{1} \\
F_{2}
\end{array}\right] \text { in }\left(V_{k, h}^{\prime}\right)^{\otimes m} .
\end{gathered}
$$

Theorem 4.4 applies to problem (6.5) as a consequence of tensor product structure (see Remark 4.5). Therefore we conclude the stability of the FTP-FE discretization $V_{k, h}^{\otimes m}$.

Let $M_{s}^{\otimes m}=\mathcal{M}^{m}\left[\begin{array}{l}u \\ p\end{array}\right]$ be the unique solution of problem (4.5) and $M_{s, h}^{\otimes m}$ be the unique solution of problem (6.5). Exploiting Galerkin orthogonality and the stability of the discretization, we can obtain the following quasi-optimal convergence estimate:

$$
\left\|\mathcal{M}^{m}\left[\begin{array}{l}
u \\
p
\end{array}\right]-M_{s, h}^{\otimes m}\right\|\left\|_{V_{k}^{\otimes m}} \leqslant C \inf _{M_{h}^{\otimes m} \in V_{k, h}^{\otimes m}}\right\| \mathcal{M}^{m}\left[\begin{array}{l}
u \\
p
\end{array}\right]-M_{h}^{\otimes m} \|_{V_{k}^{\otimes m}} .
$$

To study the approximation properties of the space $V_{k, h}^{\otimes m}$ we construct the tensorial projection operator $\Pi_{\mathbf{k}, h}^{\otimes m}, \mathbf{k}=\left(k_{1}, \ldots, k_{m}\right)$, as follows.

Definition 6.2 Let $\Pi_{k, h}: H_{\Gamma_{\mathrm{D}}} \Lambda^{k}(D) \rightarrow \mathcal{P}_{r, \Gamma_{\mathrm{D}}}^{-} \Lambda^{k}\left(\mathcal{T}_{h}\right)$ be a bounded cochain projector satisfying Assumption 6.1. Given $m \geqslant 2$ integer, we define the tensor product operator mapping $H_{\Gamma_{\mathrm{D}}} \Lambda^{k_{1}}(D) \otimes$ $\cdots \otimes H_{\Gamma_{\mathrm{D}}} \Lambda^{k_{m}}(D)$ onto $\mathcal{P}_{r, \Gamma_{\mathrm{D}}}^{-} \Lambda^{k_{1}}\left(\mathcal{T}_{h}\right) \otimes \cdots \otimes \mathcal{P}_{r, \Gamma_{\mathrm{D}}}^{-} \Lambda^{k_{m}}\left(\mathcal{T}_{h}\right)$ as

$$
\Pi_{\mathbf{k}, h}^{\otimes m}:=\Pi_{k_{1}, h} \otimes \cdots \otimes \Pi_{k_{m}, h}, \quad \mathbf{k}=\left(k_{1}, \ldots, k_{m}\right) .
$$

Note that $\Pi_{\mathbf{k}, h}^{\otimes m}=\left(\Pi_{k, h}\right)^{\otimes m}=\Pi_{k, h}^{\otimes m}$ if $\mathbf{k}=(k, \ldots, k)$. In the following we denote $\Pi_{\mathbf{k}, h}^{\otimes m}$ as $\Pi_{h}^{\otimes m}$ when no ambiguity arises.

Since $\Pi_{h}$ is bounded in the $H \Lambda^{k}$ norm by a constant which we denote by $C_{\pi}$, then $\Pi_{h}^{\otimes m}$ is bounded in the $\left(H \Lambda^{k_{1}} \otimes \cdots \otimes H \Lambda^{k_{m}}\right)$ norm by $\left(C_{\pi}\right)^{m}$ (Proposition 4.1). Moreover, since it is the tensor product of cochain projectors, it is itself a cochain projector.

We state the approximation properties of $\Pi_{h}^{\otimes m}$ in the following proposition. 
Proposition 6.3 The projector $\Pi_{h}^{\otimes m}$ introduced in Definition 6.2 is such that

$$
\left\|v-\Pi_{h}^{\otimes m} v\right\|_{\left(L^{2} \Lambda^{k}\right)^{\otimes m}} \leqslant C h^{s}\|v\|_{\left(H^{s} \Lambda^{k}\right) \otimes m}
$$

for all $v \in\left(H^{s} \Lambda^{k}(D) \cap H_{\Gamma_{\mathrm{D}}} \Lambda^{k}(D)\right)^{\otimes m}, 0 \leqslant s \leqslant r$, where $C$ is independent of $h$.

Proof. We already know the result for $m=1$ (see (6.2)). Let $m=2$. By the triangle inequality,

$$
\begin{aligned}
\left\|v-\Pi_{h}^{\otimes 2} v\right\|_{L^{2} \Lambda^{k} \otimes L^{2} \Lambda^{k}} & \leqslant\left\|v-\Pi_{h} \otimes \operatorname{Id} v\right\|_{L^{2} \Lambda^{k} \otimes L^{2} \Lambda^{k}}+\left\|\Pi_{h} \otimes\left(\mathrm{Id}-\Pi_{h}\right) v\right\|_{L^{2} \Lambda^{k} \otimes L^{2} \Lambda^{k}} \\
& \leqslant C h^{s}\|v\|_{H^{s} \Lambda^{k} \otimes L^{2} \Lambda^{k}}+C_{\pi}\left\|v-\operatorname{Id} \otimes \Pi_{h} v\right\|_{L^{2} \Lambda^{k} \otimes L^{2} \Lambda^{k}} \\
& \leqslant C h^{s}\|v\|_{H^{s} \Lambda^{k} \otimes L^{2} \Lambda^{k}}+C C_{\pi} h^{s}\|v\|_{L^{2} \Lambda^{k} \otimes H^{s} \Lambda^{k}} \\
& \leqslant C h^{s}\left(1+C_{\pi}\right)\|v\|_{H^{s} \Lambda^{k} \otimes H^{s} \Lambda^{k}}
\end{aligned}
$$

where we used (6.2). By induction on $m$, we conclude (6.8).

From the approximation properties of the projector $\Pi_{h}^{\otimes m}$ in (6.8), we obtain the following theorem. THEOREM 6.4 (Order of convergence of the FTP-FE discretization.). We have

$$
\left\|\mathcal{M}^{m}\left[\begin{array}{l}
u \\
p
\end{array}\right]-M_{s, h}^{\otimes m}\right\|_{V_{k}^{\otimes m}}=\mathcal{O}\left(h^{r}\right),
$$

provided that

$$
\begin{gathered}
{\left[\begin{array}{l}
u \\
p
\end{array}\right] \in L^{m}\left(\Omega ;\left[\begin{array}{c}
H^{r} \Lambda^{k}(D) \cap H_{\Gamma_{\mathrm{D}}} \Lambda^{k}(D) \\
H^{r} \Lambda^{k-1}(D) \cap H_{\Gamma_{\mathrm{D}}} \Lambda^{k-1}(D)
\end{array}\right]\right),} \\
{\left[\begin{array}{l}
\mathrm{d} u \\
\mathrm{~d} p
\end{array}\right] \in L^{m}\left(\Omega ;\left[\begin{array}{c}
H^{r} \Lambda^{k+1}(D) \cap H \Lambda^{k+1}(D) \\
H^{r} \Lambda^{k}(D) \cap H \Lambda^{k}(D)
\end{array}\right]\right) .}
\end{gathered}
$$

\subsection{Discrete mth moment problem: sparse tensor product approximation}

In Section 6.2, we proved the stability of the FTP-FE discretization $V_{k, h}^{\otimes m}=\underbrace{V_{k, h} \otimes \cdots \otimes V_{k, h}}_{m \text { times }}$. The main problem of this approach is that it is strongly affected by the curse of dimensionality. Indeed, if $\operatorname{dim}\left(V_{k, h}\right)=N_{h}$, the space $V_{k, h}^{\otimes m}$ has dimension $\left(N_{h}\right)^{m}$ which is impractical for $m$ moderately large. A reduction in the dimensionality of the problem is possible if we consider an STP-FE approximation instead (see e.g. Schwab \& Todor, 2003; Bungartz \& Griebel, 2004; von Petersdorff \& Schwab, 2006; Harbrecht et al., 2008b; Schwab \& Gittelson, 2011 and the references therein).

Let $\mathcal{T}_{0}$ be a regular mesh of the physical domain $D \subset \mathbb{R}^{n}$, and $\left\{\mathcal{T}_{l}\right\}_{l=0}^{\infty}$ be a sequence of partitions obtained by uniform mesh refinement, that is, $h_{l}=h_{l-1} / 2$, where $h_{l}$ is the discretization parameter of $\mathcal{T}_{l}$. We have a sequence $\left\{\mathcal{P}_{r}^{-} \Lambda^{k}\left(\mathcal{T}_{l}\right)\right\}_{l=0}^{\infty}$ of finite-dimensional subspaces of the space $V_{k}$, which are nested and dense in $V_{k}$. Let us define the orthogonal complement of $\mathcal{P}_{r}^{-} \Lambda^{k}\left(\mathcal{T}_{l-1}\right)$ in $\mathcal{P}_{r}^{-} \Lambda^{k}\left(\mathcal{T}_{l}\right): S_{k, l}=$ $\mathcal{P}_{r}^{-} \Lambda^{k}\left(\mathcal{T}_{l}\right) \backslash \mathcal{P}_{r}^{-} \Lambda^{k}\left(\mathcal{T}_{l-1}\right)$ and set $Z_{k, l}=\left[\begin{array}{c}S_{k, l} \\ S_{k-1, l}\end{array}\right]$. For every integer $m \geqslant 2$, we define the STP-FE space 
of level $L>0, V_{\mathbf{k}, L}^{(m)}$, as follows:

$$
V_{\mathbf{k}, L}^{(m)}:=\bigoplus_{|\mathbf{l}| \leqslant L}\left(Z_{k_{1}, l_{1}} \otimes \cdots \otimes Z_{k_{m}, l_{m}}\right), \quad \mathbf{k}=\left(k_{1}, \ldots, k_{m}\right),
$$

where $\mathbf{l}$ is a multiindex in $\mathbb{N}_{0}^{m}$ and $|\mathbf{I}|$ is its length $l_{1}+\cdots+l_{m}$. If $\mathbf{k}=(k, \ldots, k)$, we denote the space (6.10) as $V_{k, L}^{(m)}$.

At the numerical level it may not be necessary to explicitly build a basis for $Z_{k, l}$. In Harbrecht et al. (2008a), the authors propose to use a redundant basis for the space (6.10) and an algorithm to solve the $m$ th moment problem in the sparse tensor product framework.

The STP-FE approximation of problem (4.5) is as follows.

\section{$m$-Points Correlation Problem (STP-FE)}

$$
\begin{gathered}
\text { Given } m \geqslant 2 \text { integer and }\left[\begin{array}{l}
F_{1} \\
F_{2}
\end{array}\right] \in L^{m}\left(\Omega ; V_{k}^{\prime}\right) \text {, find } M_{s, L}^{(m)} \in V_{k, L}^{(m)} \text { such that } \\
T^{\otimes m} M_{s, L}^{(m)}=\mathcal{M}^{m}\left[\begin{array}{l}
F_{1} \\
F_{2}
\end{array}\right] \quad \text { in }\left(V_{k, L}^{(m)}\right)^{\prime} .
\end{gathered}
$$

To prove the stability of (6.11) we cannot use a tensor product argument as we did to prove the stability of the FTP-FE discretization. We need to explicitly prove the inf-sup condition for the tensor product operator $T^{\otimes m}$ restricted to the STP-FE space $V_{k, L}^{(m)}$. The proof of this sparse inf-sup condition rests on two key ingredients. On the one hand, we make use of the continuous inf-sup operator $P^{\otimes m}$ introduced in Theorem 4.6. On the other hand, we use a reasoning similar to the one proposed in Buffa (2005) which defines and uses the so-called GAP property: we seek its analogue in the case of STP-FE space, which will be called the STP-GAP property in what follows. The main ingredient of the STP-GAP property is the sparse tensorial projection operator.

DEFINITION 6.5 Let $\Pi_{k, h}: H_{\Gamma_{\mathrm{D}}} \Lambda^{k}(D) \rightarrow \mathcal{P}_{r, \Gamma_{\mathrm{D}}}^{-} \Lambda^{k}\left(\mathcal{T}_{h}\right)$ be a bounded cochain projector satisfying Assumption 6.1. Given $m \geqslant 2$ integer, we define the operator mapping $H_{\Gamma_{\mathrm{D}}} \Lambda^{k_{1}}(D) \otimes \cdots \otimes H_{\Gamma_{\mathrm{D}}} \Lambda^{k_{m}}(D)$ onto $\bigoplus_{|\boldsymbol{I}| \leqslant L}\left(S_{k, l_{1}} \otimes \cdots \otimes S_{k, l_{m}}\right)$ as

$$
\Pi_{\mathbf{k}, L}^{(m)}:=\sum_{|\mathbf{1}| \leqslant L} \otimes \Delta_{k_{j}, l_{j}}, \quad \mathbf{k}=\left(k_{1}, \ldots, k_{m}\right)
$$

where $\Delta_{k, l}:=\Pi_{k, h_{l}}-\Pi_{k, h_{l-1}}$.

With a little abuse of notation, in what follows we omit the subscript $\mathbf{k}$ and denote the operator (6.12) by $\Pi_{L}^{(m)}$, and $\Delta_{k, l}$ by $\Delta_{l}$.

The operator $\Pi_{L}^{(m)}$ is a linear combination of the tensor product operators $\Delta_{l_{1}} \otimes \cdots \otimes \Delta_{l_{m}}$. Since each $\Delta_{l}$ is bounded, then $\Delta_{l_{1}} \otimes \cdots \otimes \Delta_{l_{m}}$ is bounded owing to Proposition 4.1, so that $\Pi_{L}^{(m)}$ is bounded. Moreover, since each $\Delta_{l}$ is a cochain operator (it commutes with the exterior derivative d), then $\Delta_{l_{1}} \otimes$ $\cdots \otimes \Delta_{l_{m}}$ is a cochain operator in the sense that it commutes with $\mathrm{d}$ in each direction $j=1, \ldots, m$, so that $\Pi_{L}^{(m)}$ is a cochain operator. Finally, the following general result states that $\Pi_{L}^{(m)}$ is a projector. We refer the reader to Delvos (1982), Novak \& Ritter (1996) and Bäck et al. (2011, Proposition 1(b)). 
Proposition 6.6 For each direction $d=1, \ldots, m$, let $W_{d}$ be a separable Hilbert space and

$$
W_{d, 0} \subset W_{d, 1} \subset \cdots \subset W_{d, l} \subset \cdots \subset W_{d}
$$

a sequence of nested finite-dimensional subspaces of $W_{d}$. Moreover, let $P_{d, l}: W_{d} \rightarrow W_{d, l}$ be a sequence of operators that are projectors on $W_{d, l}$ for all $l=0,1, \ldots$, and $P_{d,-1}=0$. Then, for all positive integers $L$ and $m$, the operator

$$
P_{L}^{(m)}:=\sum_{\left|\left(l_{1}, \ldots, l_{m}\right)\right| \leqslant L} \Delta P_{1, l_{1}} \otimes \cdots \otimes \Delta P_{m, l_{m}}
$$

is a projector on the space

$$
W_{L}^{(m)}:=\sum_{\left|\left(l_{1}, \ldots, l_{m}\right)\right| \leqslant L} W_{1, l_{1}} \otimes \cdots \otimes W_{m, l_{m}},
$$

where $\Delta P_{d, l}:=P_{d, l}-P_{d, l-1}, d=1, \ldots, m$.

Proof. Since the operator $P_{L}^{(m)}$ is linear, we only need to prove the result for an element of $W_{L}^{(m)}$ of the form $w=\psi_{j_{1}} \otimes \cdots \otimes \psi_{j_{m}} \in W_{1, j_{1}} \otimes \cdots \otimes W_{m, j_{m}}$, where $\left|\left(j_{1}, \ldots, j_{m}\right)\right| \leqslant L$. We have

$$
\begin{aligned}
P_{L}^{(m)}(w) & =\sum_{\left|\left(l_{1}, \ldots, l_{m}\right)\right| \leqslant L} \Delta P_{1, l_{1}} \otimes \cdots \otimes \Delta P_{m, l_{m}}(w) \\
& =\sum_{\left|\left(l_{1}, \ldots, l_{m}\right)\right| \leqslant L} \Delta P_{1, l_{1}} \otimes \cdots \otimes \Delta P_{m, l_{m}}\left(\psi_{j_{1}} \otimes \cdots \otimes \psi_{j_{m}}\right) \\
& =\sum_{\left|\left(l_{1}, \ldots, l_{m}\right)\right| \leqslant L} \Delta P_{1, l_{1}}\left(\psi_{j_{1}}\right) \otimes \cdots \otimes \Delta P_{m, l_{m}}\left(\psi_{j_{m}}\right) .
\end{aligned}
$$

Since $P_{d, l}\left(\psi_{j}\right)=\psi_{j}$ whenever $l \geqslant j$, then $\Delta P_{d, l}\left(\psi_{j}\right)=0$ for $l \geqslant j+1, d=1, \ldots, m$. Hence,

$$
\begin{aligned}
(6.13) & =\sum_{\left(l_{1}, \ldots, l_{m}\right) \leqslant\left(j_{1}, \ldots, j_{m}\right)} \Delta P_{1, l_{1}}\left(\psi_{j_{1}}\right) \otimes \cdots \otimes \Delta P_{m, l_{m}}\left(\psi_{j_{m}}\right) \\
& =\left(\sum_{l_{1}=0}^{j_{1}}\left(P_{1, l_{1}}-P_{1, l_{1}-1}\right)\left(\psi_{j_{1}}\right)\right) \otimes \cdots \otimes\left(\sum_{l_{m}=0}^{j_{m}}\left(P_{m, l_{m}}-P_{m, l_{m}-1}\right)\left(\psi_{j_{m}}\right)\right) \\
& =P_{1, j_{1}}\left(\psi_{j_{1}}\right) \otimes \cdots \otimes P_{m, j_{m}}\left(\psi_{j_{m}}\right) \\
& =\psi_{j_{1}} \otimes \cdots \otimes \psi_{j_{m}}=w
\end{aligned}
$$

where we used that $P_{d, l}$ is a projector on $V_{d, l}, d=1, \ldots, m$.

We state the STP-GAP property for $m=2$, but its generalization to $m \geqslant 2$ is straightforward.

LEMMA 6.7 (STP-GAP property) For every $v_{h} \in \Pi_{L}^{(2)}\left(H_{\Gamma_{\mathrm{D}}} \Lambda^{k}(D) \otimes H_{\Gamma_{\mathrm{D}}} \Lambda^{k}(D)\right)$ there exist $0<s \leqslant 1$ and positive constants $C^{(1)}, C^{(2)}, C^{(3)}, C^{(4)}$ independent of $h_{0}$ such that

$$
\left\|\mathrm{d} \pi^{\circ} \otimes \mathrm{d} \pi^{\circ} v_{h}-\Pi_{L}^{(2)}\left(\mathrm{d} \pi^{\circ} \otimes \mathrm{d} \pi^{\circ} v_{h}\right)\right\|_{H \Lambda^{k} \otimes H \Lambda^{k}} \leqslant C^{(1)} h_{0}^{s}\left\|v_{h}\right\|_{H \Lambda^{k} \otimes H \Lambda^{k}},
$$




$$
\begin{gathered}
\left\|\mathrm{d} \pi^{\circ} \otimes \pi^{\perp} v_{h}-\Pi_{L}^{(2)}\left(\mathrm{d} \pi^{\circ} \otimes \pi^{\perp} v_{h}\right)\right\|_{H \Lambda^{k} \otimes H \Lambda^{k}} \leqslant C^{(2)} h_{0}^{s}\left\|v_{h}\right\|_{H \Lambda^{k} \otimes H \Lambda^{k}}, \\
\left\|\pi^{\perp} \otimes \mathrm{d} \pi^{\circ} v_{h}-\Pi_{L}^{(2)}\left(\pi^{\perp} \otimes \mathrm{d} \pi^{\circ} v_{h} t\right)\right\|_{H \Lambda^{k} \otimes H \Lambda^{k}} \leqslant C^{(3)} h_{0}^{s}\left\|v_{h}\right\|_{H \Lambda^{k} \otimes H \Lambda^{k}}, \\
\left\|\pi^{\perp} \otimes \pi^{\perp} v_{h}-\Pi_{L}^{(2)}\left(\pi^{\perp} \otimes \pi^{\perp} v_{h}\right)\right\|_{H \Lambda^{k} \otimes H \Lambda^{k}} \leqslant C^{(4)} h_{0}^{s}\left\|v_{h}\right\|_{H \Lambda^{k} \otimes H \Lambda^{k}},
\end{gathered}
$$

where $\pi^{\perp}$ and $\pi^{\circ}$ are defined in (2.10) and (2.11), respectively. Note that $v_{h}$ is uniquely expressed as $v_{h}=\mathrm{d} \pi^{\circ} \otimes \mathrm{d} \pi^{\circ} v_{h}+\mathrm{d} \pi^{\circ} \otimes \pi^{\perp} v_{h}+\pi^{\perp} \otimes \mathrm{d} \pi^{\circ} v_{h}+\pi^{\perp} \otimes \pi^{\perp} v_{h}$ owing to the continuous Hodge decomposition (4.12).

Proof. Let $v_{h} \in \Pi_{L}^{(2)}\left(H_{\Gamma_{\mathrm{D}}} \Lambda^{k}(D) \otimes H_{\Gamma_{\mathrm{D}}} \Lambda^{k}(D)\right)$, so that $\Pi_{L}^{(2)} v_{h}=v_{h}$. Since $\Pi_{L}^{(2)}$ is a cochain map, it holds that

$$
\begin{gathered}
\mathrm{d} \otimes \mathrm{d} v_{h}=\mathrm{d} \otimes \mathrm{d} \Pi_{L}^{(2)} v_{h}=\Pi_{L}^{(2)} \mathrm{d} \otimes \mathrm{d} v_{h}, \\
\mathrm{~d} \otimes \mathrm{Id} v_{h}=\mathrm{d} \otimes \mathrm{Id} \Pi_{L}^{(2)} v_{h}=\Pi_{L}^{(2)} \mathrm{d} \otimes \mathrm{Id} v_{h}, \\
\mathrm{Id} \otimes \mathrm{d} v_{h}=\mathrm{Id} \otimes \mathrm{d} \Pi_{L}^{(2)} v_{h}=\Pi_{L}^{(2)} \mathrm{Id} \otimes \mathrm{d} v_{h} .
\end{gathered}
$$

By definition of $\mathfrak{B}_{k}^{\perp}$ and Assumption 2.2, $\mathfrak{B}_{k}^{\perp} \subset H_{\Gamma_{\mathrm{D}}} \Lambda^{k} \cap H_{\Gamma_{\mathrm{N}}}^{*} \Lambda^{k}$, so that, owing to Assumption 2.3,

$$
\left\|\Delta_{l} w\right\|_{L^{2} \Lambda^{k}} \leqslant C h_{l-1}^{s}\|w\|_{H^{s} \Lambda^{k}} \leqslant \tilde{C} h_{l-1}^{s}\|w\|_{H \Lambda^{k}} \quad \forall w \in \mathfrak{B}_{k}^{\perp} .
$$

- Let us start by proving inequality (6.17). To this end, we need to bound four quantities:

$$
\begin{aligned}
& \left\|\pi^{\perp} \otimes \pi^{\perp} v_{h}-\Pi_{L}^{(2)}\left(\pi^{\perp} \otimes \pi^{\perp} v_{h}\right)\right\|_{L^{2} \Lambda^{k} \otimes L^{2} \Lambda^{k},} \\
& \left\|\mathrm{~d} \pi^{\perp} \otimes \pi^{\perp} v_{h}-\Pi_{L}^{(2)}\left(\mathrm{d} \pi^{\perp} \otimes \pi^{\perp} v_{h}\right)\right\|_{L^{2} \Lambda^{k+1} \otimes L^{2} \Lambda^{k},} \\
& \left\|\pi^{\perp} \otimes \mathrm{d} \pi^{\perp} v_{h}-\Pi_{L}^{(2)}\left(\pi^{\perp} \otimes \mathrm{d} \pi^{\perp} v_{h}\right)\right\|_{L^{2} \Lambda^{k} \otimes L^{2} \Lambda^{k+1},} \\
& \left\|\mathrm{~d} \pi^{\perp} \otimes \mathrm{d} \pi^{\perp} v_{h}-\Pi_{L}^{(2)}\left(\mathrm{d} \pi^{\perp} \otimes \mathrm{d} \pi^{\perp} v_{h}\right)\right\|_{L^{2} \Lambda^{k+1} \otimes L^{2} \Lambda^{k+1}} .
\end{aligned}
$$

Using the fact that $\pi^{\perp} \otimes \pi^{\perp} v_{h}=\sum_{L=0}^{+\infty} \sum_{|\mathbf{I}|=L} \Delta_{l_{1}} \otimes \Delta_{l_{2}} v_{h}$, the triangle inequality and (6.21),

$$
\begin{aligned}
(6.22) & \leqslant \sum_{|\mathbf{|}|>L}\left\|\left(\Delta_{l_{1}} \otimes \Delta_{l_{2}}\right)\left(\pi^{\perp} \otimes \pi^{\perp}\right) v_{h}\right\|_{L^{2} \Lambda^{k} \otimes L^{2} \Lambda^{k}} \\
& =\sum_{|\mathbf{I}|>L}\left\|\left(\Delta_{l_{1}} \pi^{\perp} \otimes \operatorname{Id}\right)\left(\operatorname{Id} \otimes \Delta_{l_{2}} \pi^{\perp}\right) v_{h}\right\|_{L^{2} \Lambda^{k} \otimes L^{2} \Lambda^{k}} \\
& \leqslant \sum_{|\mathbf{|}|>L} C h_{l_{1}-1}^{s}\left\|\left(\operatorname{Id} \otimes \Delta_{l_{2}} \pi^{\perp}\right) v_{h}\right\|_{H \Lambda^{k} \otimes L^{2} \Lambda^{k}} \\
& \leqslant \sum_{|\mathbf{I}|>L} C h_{l_{1}-1}^{s} h_{l_{2}-1}^{s}\left\|v_{h}\right\|_{H \Lambda^{k} \otimes H \Lambda^{k}},
\end{aligned}
$$

where $C>0$ is independent of $h_{l}$ for all $l$. Observing that

$$
(\mathrm{d} \otimes \mathrm{Id})\left(\pi^{\perp} \otimes \pi^{\perp} v_{h}\right)=\mathrm{d} \otimes \pi^{\perp} v_{h} \in \Pi_{L}\left(H_{\Gamma_{\mathrm{D}}} \Lambda^{k}(D)\right) \otimes \mathfrak{B}_{k}^{\perp},
$$


so that $\left(\Delta_{l_{1}} \otimes \mathrm{Id}\right)\left(\mathrm{d} \otimes \pi^{\perp} v_{h}\right)=0$ if $l_{1}>L$, we can bound (6.23):

$$
\begin{aligned}
(6.23) & =\left\|\mathrm{d} \otimes \pi^{\perp} v_{h}-\Pi_{L}^{(2)}\left(\mathrm{d} \otimes \pi^{\perp} v_{h}\right)\right\|_{L^{2} \Lambda^{k+1} \otimes L^{2} \Lambda^{k}} \\
& \leqslant \sum_{l_{1}=0}^{L} \sum_{l_{2}=L-l_{1}+1}^{+\infty}\left\|\left(\Delta_{l_{1}} \otimes \Delta_{l_{2}}\right)\left(\mathrm{d} \otimes \pi^{\perp}\right) v_{h}\right\|_{L^{2} \Lambda^{k+1} \otimes L^{2} \Lambda^{k}} \\
& \leqslant \sum_{l_{1}=0}^{L} \sum_{l_{2}=L-l_{1}+1}^{+\infty}\left\|\Delta_{l_{1}}\right\|_{\mathcal{L}\left(L^{2} \Lambda^{k+1}, L^{2} \Lambda^{k+1}\right)}\left\|\left(\mathrm{Id} \otimes \Delta_{l_{2}}\right)\left(\mathrm{d} \otimes \pi^{\perp}\right) v_{h}\right\|_{L^{2} \Lambda^{k+1} \otimes L^{2} \Lambda^{k}} \\
& \leqslant C \sum_{l_{1}=0}^{L} \sum_{l_{2}=L-l_{1}+1}^{+\infty} h_{l_{2}-1}^{s}\left\|\mathrm{~d} \otimes \operatorname{Id} v_{h}\right\|_{L^{2} \Lambda^{k+1} \otimes H \Lambda^{k}} \\
& \leqslant C(L+1) \sum_{l_{2}=1}^{+\infty} h_{l_{2}-1}^{s}\left\|v_{h}\right\|_{H \Lambda^{k} \otimes H \Lambda^{k}} \\
& \leqslant C h_{0}^{s}\left\|v_{h}\right\|_{H \Lambda^{k} \otimes H \Lambda^{k},}
\end{aligned}
$$

where we have used that $\left\|\Delta_{l_{1}}\right\|_{\mathcal{L}\left(L^{2} \Lambda^{k+1}, L^{2} \Lambda^{k+1}\right)}$ is bounded by a constant independent of $h_{l_{1}}$. By symmetry, we can obtain that

$$
(6.24) \leqslant C h_{0}^{s}\left\|v_{h}\right\|_{H \Lambda^{k} \otimes H \Lambda^{k}}
$$

Finally, using (6.18), we have

$$
(\mathrm{d} \otimes \mathrm{d})\left(\pi^{\perp} \otimes \pi^{\perp}\right) v_{h}=\mathrm{d} \otimes \mathrm{d} v_{h}=\mathrm{d} \otimes \mathrm{d} \Pi_{L}^{(2)} v_{h}=\Pi_{L}^{(2)}(\mathrm{d} \otimes \mathrm{d})\left(\pi^{\perp} \otimes \pi^{\perp}\right) v_{h},
$$

so that the quantity in (6.25) vanishes. Thus, putting together (6.26-6.28), we conclude (6.17).

- Let us prove inequality (6.16). We need to bound two quantities:

$$
\begin{aligned}
& \left\|\pi^{\perp} \otimes \mathrm{d} \pi^{\circ} v_{h}-\Pi_{L}^{(2)}\left(\pi^{\perp} \otimes \mathrm{d} \pi^{\circ} v_{h}\right)\right\|_{L^{2} \Lambda^{k} \otimes L^{2} \Lambda^{k},} \\
& \left\|\mathrm{~d} \pi^{\perp} \otimes \mathrm{d} \pi^{\circ} v_{h}-\Pi_{L}^{(2)}\left(\mathrm{d} \pi^{\perp} \otimes \mathrm{d} \pi^{\circ} v_{h}\right)\right\|_{L^{2} \Lambda^{k+1} \otimes L^{2} \Lambda^{k} .}
\end{aligned}
$$

Since $\pi^{\perp} \otimes \mathrm{d} \pi^{\circ} v_{h}=\pi^{\perp} \otimes \operatorname{Id} v_{h}-\pi^{\perp} \otimes \pi^{\perp} v_{h}$ and $\pi^{\perp} \otimes \operatorname{Id} v_{h} \in \mathfrak{B}_{k}^{\perp} \otimes \Pi_{L}\left(H_{\Gamma_{\mathrm{D}}} \Lambda^{k}(D)\right)$, and using (6.17), then

$$
\begin{aligned}
(6.29) \leqslant & \left\|\pi^{\perp} \otimes \operatorname{Id} v_{h}-\Pi_{L}^{(2)} \pi^{\perp} \otimes \operatorname{Id} v_{h}\right\|_{L^{2} \Lambda^{k} \otimes L^{2} \Lambda^{k}} \\
& +\left\|\pi^{\perp} \otimes \pi^{\perp} v_{h}-\Pi_{L}^{(2)} \pi^{\perp} \otimes \pi^{\perp} v_{h}\right\|_{L^{2} \Lambda^{k} \otimes L^{2} \Lambda^{k}} \\
\leqslant & \sum_{l_{2}=0}^{L} \sum_{l_{1}=L+1-l_{2}}^{+\infty}\left\|\left(\Delta_{l_{1}} \otimes \Delta_{l_{2}}\right)\left(\pi^{\perp} \otimes \operatorname{Id}\right) v_{h}\right\|_{L^{2} \Lambda^{k} \otimes L^{2} \Lambda^{k}}+C h_{0}^{s}\left\|v_{h}\right\|_{H \Lambda^{k} \otimes H \Lambda^{k}} \\
\leqslant & \sum_{l_{2}=0}^{L} \sum_{l_{1}=L+1-l_{2}}^{+\infty}\left\|\Delta_{l_{2}}\right\|_{\mathcal{L}\left(L^{2} \Lambda^{k}, L^{2} \Lambda^{k}\right)} h_{l_{1}-1}^{s}\left\|v_{h}\right\|_{H \Lambda^{k} \otimes H \Lambda^{k}}+C h_{0}^{s}\left\|v_{h}\right\|_{H \Lambda^{k} \otimes H \Lambda^{k}} \\
\leqslant & C h_{0}^{s}\left\|v_{h}\right\|_{H \Lambda^{k} \otimes H \Lambda^{k} .}
\end{aligned}
$$


Moreover, using (6.17),

$$
\begin{aligned}
(6.30) \leqslant & \left\|\mathrm{d} \pi^{\perp} \otimes \operatorname{Id} v_{h}-\Pi_{L}^{(2)} \mathrm{d} \pi^{\perp} \otimes \operatorname{Id} v_{h}\right\|_{L^{2} \Lambda^{k+1} \otimes L^{2} \Lambda^{k}} \\
& +\left\|\mathrm{d} \pi^{\perp} \otimes \pi^{\perp} v_{h}-\Pi_{L}^{(2)} \mathrm{d} \pi^{\perp} \otimes \pi^{\perp} v_{h}\right\|_{L^{2} \Lambda^{k+1} \otimes L^{2} \Lambda^{k}} \\
\leqslant & C h_{0}^{s}\left\|v_{h}\right\|_{H \Lambda^{k} \otimes H \Lambda^{k}} .
\end{aligned}
$$

In the last inequality we exploited (6.19), which implies that $\mathrm{d} \pi^{\perp} \otimes \operatorname{Id} v_{h}=\mathrm{d} \otimes \operatorname{Id} v_{h}=\mathrm{d} \otimes$ $\operatorname{Id} \Pi_{L}^{(2)} v_{h}=\Pi_{L}^{(2)} \mathrm{d} \pi^{\perp} \otimes \operatorname{Id} v_{h}$, so that

$$
\left\|\mathrm{d} \pi^{\perp} \otimes \operatorname{Id} v_{h}-\Pi_{L}^{(2)} \mathrm{d} \pi^{\perp} \otimes \operatorname{Id} v_{h}\right\|_{L^{2} \Lambda^{k+1} \otimes L^{2} \Lambda^{k}}=0 .
$$

Using (6.31) and (6.32) we conclude (6.16).

- To show (6.15), we write $v_{h}$ as $v_{h}=\mathrm{Id} \otimes \mathrm{d} \pi^{\circ} v_{h}+\mathrm{Id} \otimes \pi^{\perp} v_{h}$ and proceed as in the proof of (6.16).

- To show (6.14) we observe that

$$
\begin{aligned}
&\left\|\mathrm{d} \pi^{\circ} \otimes \mathrm{d} \pi^{\circ} v_{h}-\Pi_{L}^{(2)}\left(\mathrm{d} \pi^{\circ} \otimes \mathrm{d} \pi^{\circ} v_{h}\right)\right\|_{H \Lambda^{k} \otimes H} \Lambda^{k} \\
&=\left\|\left(\mathrm{Id} \otimes \mathrm{Id}-\Pi_{L}^{(2)}\right)\left(\mathrm{Id} \otimes \mathrm{Id}-\mathrm{d} \pi^{\circ} \otimes \pi^{\perp}-\pi^{\perp} \otimes \mathrm{d} \pi^{\circ}-\pi^{\perp} \otimes \pi^{\perp}\right) v_{h}\right\|_{H \Lambda^{k} \otimes H} \Lambda^{k} \\
& \leqslant\left\|v_{h}-\Pi_{L}^{(2)} v_{h}\right\|_{H \Lambda^{k} \otimes H \Lambda^{k}}+\left\|\mathrm{d} \pi^{\circ} \otimes \pi^{\perp} v_{h}-\Pi_{L}^{(2)} \mathrm{d} \pi^{\circ} \otimes \pi^{\perp} v_{h}\right\|_{H \Lambda^{k} \otimes H \Lambda^{k}} \\
&+\left\|\pi^{\perp} \otimes \mathrm{d} \pi^{\circ} v_{h}-\Pi_{L}^{(2)} \pi^{\perp} \otimes \mathrm{d} \pi^{\circ} v_{h}\right\|_{H \Lambda^{k} \otimes H} \Lambda^{k} \\
&+\left\|\pi^{\perp} \otimes \pi^{\perp} v_{h}-\Pi_{L}^{(2)} \pi^{\perp} \otimes \pi^{\perp} v_{h}\right\|_{H \Lambda^{k} \otimes H} \Lambda^{k}
\end{aligned}
$$

and we conclude (6.14) using the fact that $v_{h}=\Pi_{L}^{(2)} v_{h}$ and (6.15-6.17).

We are now ready to prove the main result of this section. It deals with vector quantities in $V_{k}$. In this context, $\Pi_{L}^{(m)}$ denotes the projector from $V_{k}^{\otimes m}$ onto $V_{k, L}^{(m)}$.

THEOREM 6.8 (Stability of the STP-FE discretization) For every $\alpha \geqslant 0$ there exists $\bar{h}_{0}>0$ such that, for all $h_{0} \leqslant \bar{h}_{0}$, problem (6.11) is a stable discretization for the $m$ th moment problem (4.5). In particular, for every $M_{s, L}^{(m)} \in V_{k, L}^{(m)}$, there exist a test function $M_{t, L}^{(m)} \in V_{k, L}^{(m)}$ and positive constants $C_{m, \text { disc }}=C_{m \text {, disc }}\left(C_{m}\right)$ $\left(C_{m}\right.$ is introduced in (4.7)), $C_{m, \mathrm{disc}}^{\prime}=C_{m, \mathrm{disc}}^{\prime}\left(\alpha,\|P\|_{\mathcal{L}\left(V_{k}, V_{k}\right)},\left\|\Pi_{L}^{(m)}\right\|_{\mathcal{L}\left(V_{k}^{\otimes m}, V_{k, L}^{(m)}\right)}\right)$ such that

$$
\begin{aligned}
\left\langle T^{\otimes m} M_{s, L}^{(m)}, M_{t, L}^{(m)}\right\rangle_{\left(V_{k, L}^{(m)}\right)^{\prime}, V_{k, L}^{(m)}} & \geqslant C_{m, \mathrm{disc}}\left\|M_{s, L}^{(m)}\right\|_{V_{k}^{\otimes m}}^{2}, \\
\left\|M_{t, L}^{(m)}\right\|_{V_{k}^{\otimes m}} & \leqslant C_{m, \mathrm{disc}}^{\prime}\left\|M_{s, L}^{(m)}\right\|_{V_{k}^{\otimes m}} .
\end{aligned}
$$

Proof. Suppose $\alpha>0$ (the case $\alpha=0$ is analogous). We fix $M_{s, L}^{(m)} \in V_{k, L}^{(m)}$ and look for a sparse test function $M_{t, L}^{(m)} \in V_{k, L}^{(m)}$ such that (6.33) and (6.34) are satisfied. We choose $M_{t, L}^{(m)}=\Pi_{L}^{(m)} P^{\otimes m} M_{s, L}^{(m)}$. Owing to Proposition 4.1 and the boundness of the operators $P$ and $\Pi_{L}^{(m)}$, we immediately conclude (6.34). In the proof of (6.33), we use brackets $\langle\cdot, \cdot\rangle$ without specifying the spaces taken into account, when no ambiguity arises. 
We have

$$
\begin{aligned}
\left\langle T^{\otimes m} M_{s, L}^{(m)}, M_{t, L}^{(m)}\right\rangle & =\left\langle T^{\otimes m} M_{s, L}^{(m)}, \Pi_{L}^{(m)} P^{\otimes m} M_{s, L}^{(m)}\right\rangle \\
& =\left\langle T^{\otimes m} M_{s, L}^{(m)}, P^{\otimes m} M_{s, L}^{(m)}\right\rangle-\left\langle T^{\otimes m} M_{s, L}^{(m)},\left(\mathrm{Id}^{\otimes m}-\Pi_{L}^{(m)}\right) P^{\otimes m} M_{s, L}^{(m)}\right\rangle .
\end{aligned}
$$

We observe that, owing to the continuous inf-sup condition (4.7),

$$
\left\langle T^{\otimes m} M_{s, L}^{(m)}, P^{\otimes m} M_{s, L}^{(m)}\right\rangle \geqslant C_{m}\left\|M_{s, L}^{(m)}\right\|_{V_{k}^{\otimes m}}^{2},
$$

and, from Lemma 6.7,

$$
\begin{aligned}
& \left\langle T^{\otimes m} M_{s, L}^{(m)},\left(\mathrm{Id}^{\otimes m}-\Pi_{L}^{(m)}\right) P^{\otimes m} M_{s, L}^{(m)}\right\rangle \\
& \quad \leqslant\|T\|_{\mathcal{L}\left(V_{k}, V_{k}^{\prime}\right)}^{m}\left\|M_{s, L}^{(m)}\right\|_{V_{k}^{\otimes m}}\left\|\left(\mathrm{Id}^{\otimes m}-\Pi_{L}^{(m)}\right) P^{\otimes m} M_{s, L}^{(m)}\right\|_{V_{k}^{\otimes m}} \\
& \quad \leqslant C h_{0}^{s}\|T\|_{\mathcal{L}\left(V_{k}, V_{k}^{\prime}\right)}^{m}\left\|M_{s, L}^{(m)}\right\|_{V_{k}^{\otimes m}}^{2} .
\end{aligned}
$$

Therefore, for $h_{0}$ sufficiently small, (6.33) follows.

REMARK 6.9 Note that the choice of the set of multiindexes $\mathcal{I}=\left\{\mathbf{I} \in \mathbb{N}^{m}:|\mathbf{I}| \leqslant L\right\}$ is not the only possibility in (6.10). Indeed, with the same techniques used in the proof of Theorem 6.8 it is possible to prove the stability of the sparse approximation in any

$$
V_{\mathbf{k}, L}^{(m)}:=\bigoplus_{\mathbf{l} \in \Lambda(L)} Z_{k_{1}, l_{1}} \otimes \cdots \otimes Z_{k_{m}, l_{m}}, \quad \mathbf{k}=\left(k_{1}, \ldots, k_{m}\right)
$$

where $\Lambda(L) \subset \mathbb{N}^{m}$ is an arbitrary index set satisfying the monotonicity property

$$
\mathbf{l} \in \Lambda(L) \rightarrow \mathbf{k} \in \Lambda(L) \quad \forall \mathbf{k} \leqslant \mathbf{l}
$$

Let $\mathcal{M}^{m}\left[\begin{array}{l}u \\ p\end{array}\right]$ be the unique solution of problem (4.5) and $M_{s, L}^{(m)}$ be the unique solution of problem (6.11). Exploiting Galerkin orthogonality and the stability of the discretization, we can obtain the following quasi-optimal convergence estimate:

$$
\left\|\mathcal{M}^{m}\left[\begin{array}{l}
u \\
p
\end{array}\right]-M_{s, L}^{(m)}\right\|_{V_{k}^{\otimes m}} \leqslant C \inf _{M_{t, L}^{(m)} \in V_{k, L}^{(m)}}\left\|\mathcal{M}^{m}\left[\begin{array}{l}
u \\
p
\end{array}\right]-M_{t, L}^{(m)}\right\|_{V_{k}^{\otimes m}}
$$

To state the approximation properties of the sparse projector $\Pi_{L}^{(m)}$ and, as a consequence, of the sparse space $V_{k, L}^{(m)}$ we need the following technical lemma.

LEMMA 6.10 It holds that

$$
\sum_{|\mathbf{I}|>L} 2^{-\gamma|\mathbf{|}|}=\sum_{i=0}^{m-1}\left(\frac{1}{2^{\gamma}-1}\right)^{m-i}\left(\begin{array}{c}
L+m \\
i
\end{array}\right) 2^{-\gamma L} \leqslant\left(\frac{1}{1-2^{-\lambda \gamma}}\right)^{m} 2^{-L \gamma(1-\lambda)}
$$

for every real $\gamma>0$ and integer $L>0$, with $0<\lambda<1$. 
Proof. The equality in (6.37) is proved in Bungartz \& Griebel (2004, Lemma 3.7). An alternative inequality to (6.37) is obtained in Bungartz \& Griebel (2004, Lemma 3.7), which, however, holds only for $\gamma \in \mathbb{N}$. Let us show the inequality in (6.37) with $\gamma>0$. Let $0<\lambda<1$; then

$$
\begin{aligned}
\sum_{i=0}^{m-1}\left(\frac{1}{2^{\gamma}-1}\right)^{m-i}\left(\begin{array}{c}
L+m \\
i
\end{array}\right) 2^{-\gamma L} & \leqslant \sum_{i=0}^{m-1}\left(\frac{1}{2^{\lambda \gamma}-1}\right)^{m-i}\left(\begin{array}{c}
L+m \\
i
\end{array}\right) 2^{-\gamma L} \\
& =\frac{2^{-\gamma L}}{\left(2^{\lambda \gamma}-1\right)^{m}} \sum_{i=0}^{m-1}\left(2^{\lambda \gamma}-1\right)^{i}\left(\begin{array}{c}
L+m \\
i
\end{array}\right) \\
& \leqslant \frac{2^{-\gamma L}}{\left(2^{\lambda \gamma}-1\right)^{m}}\left(2^{\lambda \gamma}\right)^{L+m} \\
& =\left(\frac{1}{1-2^{-\lambda \gamma}}\right)^{m} 2^{-L \gamma(1-\lambda)}
\end{aligned}
$$

REMARK 6.11 By a minimization strategy in (6.37), we derive the value of the optimal $\lambda, \lambda^{\star}=$ $(1 / \gamma) \log _{2}(m / L+1)$, so that $\sum_{|\mathbf{I}|>L} 2^{-\gamma|\boldsymbol{I}|} \leqslant(1+L / m)^{m} e^{m} 2^{-L \gamma}$. The condition $\lambda^{\star}<1$ is satisfied if and only if $L>m /\left(2^{\gamma}-1\right)$.

Proposition 6.12 The projector $\Pi_{L}^{(m)}$ introduced in Definition 6.5 is such that

$$
\left\|v-\Pi_{L}^{(m)} v\right\|_{\left(L^{2} \Lambda^{k}\right) \otimes m} \leqslant C h_{L}^{s(1-\lambda)}\|v\|_{\left(H^{s} \Lambda^{k}\right)^{\otimes m}},
$$

$0<\lambda<1$, for all $v \in\left(H_{\Gamma_{\mathrm{D}}}^{s} \Lambda^{k}(D)\right)^{\otimes m}, 0<s \leqslant r$, where $C=C(m, \lambda, s)$ is independent of $h_{L}$.

Proof. Following Bungartz \& Griebel (2004), we proceed in three steps. We start by considering the approximation properties of $\Delta_{l}$. Using the triangle inequality and (6.2) we have

$$
\left\|\Delta_{l} \otimes \mathrm{Id}^{\otimes(m-1)} v\right\|_{\left(L^{2} \Lambda^{k}\right)^{\otimes m}} \leqslant C h_{l-1}^{s}\|v\|_{H^{s} \Lambda^{k} \otimes\left(L^{2} \Lambda^{k}\right)^{\otimes(m-1)}}
$$

for every $0<s \leqslant r$. Now we consider the tensor product $\bigotimes_{j=1}^{m} \Delta_{l_{j}}$. By recursion,

$$
\left\|\bigotimes_{j=1}^{m} \Delta_{l_{j}} v\right\|_{\left(L^{2} \Lambda^{k}\right)^{\otimes m}} \leqslant C h_{\mathbf{l}-1}^{s}\|v\|_{\left(H^{s} \Lambda^{k}\right)^{\otimes m}},
$$

where $h_{\mathbf{1}-1}^{s}=h_{l_{1}-1}^{s} \cdots h_{l_{m}-1}^{s}$. Finally, using (6.10),

$$
\begin{aligned}
\left\|v-\Pi_{L}^{(m)} v\right\|_{\left(L^{2} \Lambda^{k}\right)^{\otimes m}} & =\left\|\sum_{|\mathbf{l}|>L} \bigotimes_{j=1}^{m} \Delta_{l_{j}} v\right\|_{\left(L^{2} \Lambda^{k}\right)^{\otimes m}} \leqslant \sum_{|\mathbf{l}|>L}\left\|\bigotimes_{j=1}^{m} \Delta_{l_{j}} v\right\|_{\left(L^{2} \Lambda^{k}\right)^{\otimes m}} \\
& \leqslant \sum_{|\mathbf{l}|>L} C h_{\mathbf{l}-1}^{s}\|v\|_{\left(H^{s} \Lambda^{k}\right)^{\otimes m}}
\end{aligned}
$$




$$
\begin{aligned}
& =C\|v\|_{\left(H^{s} \Lambda^{k}\right) \otimes m} h_{0}^{s m} \sum_{|\mathbf{I}|>L} 2^{-s|\mathbf{|}-1|}=C\|v\|_{\left(H^{s} \Lambda^{k}\right) \otimes m} h_{0}^{s m} 2^{s m} \sum_{|\mathbf{|}|>L} 2^{-s|\mathbf{|}|} \\
& \leqslant C\|v\|_{\left(H^{s} \Lambda^{k}\right) \otimes m} h_{0}^{s m} 2^{s m} 2^{-L s(1-\lambda)}\left(\frac{1}{1-2^{-s \lambda}}\right)^{m} \\
& =C\|v\|_{\left(H^{s} \Lambda^{k}\right)^{\otimes m}}\left(\frac{2^{s} h_{0}^{s}}{1-2^{-s \lambda}}\right)^{m} 2^{-L s(1-\lambda)}
\end{aligned}
$$

for every $0<s \leqslant r$.

We obtain the following theorem.

THEOREM 6.13 (Order of convergence of the STP-FE discretization.). We have

$$
\left\|\mathcal{M}^{m}\left[\begin{array}{l}
u \\
p
\end{array}\right]-M_{s, L}^{(m)}\right\|_{V_{k}^{\otimes m}}=\mathcal{O}\left(h_{L}^{r(1-\lambda)}\right),
$$

$0<\lambda<1$, provided that

$$
\begin{gathered}
{\left[\begin{array}{l}
u \\
p
\end{array}\right] \in L^{m}\left(\Omega ;\left[\begin{array}{c}
H^{r} \Lambda^{k}(D) \cap H_{\Gamma_{\mathrm{D}}} \Lambda^{k}(D) \\
H^{r} \Lambda^{k-1}(D) \cap H_{\Gamma_{\mathrm{D}}} \Lambda^{k-1}(D)
\end{array}\right]\right),} \\
{\left[\begin{array}{l}
\mathrm{d} u \\
\mathrm{~d} p
\end{array}\right] \in L^{m}\left(\Omega ;\left[\begin{array}{c}
H^{r} \Lambda^{k+1}(D) \cap H \Lambda^{k+1}(D) \\
H^{r} \Lambda^{k}(D) \cap H \Lambda^{k}(D)
\end{array}\right]\right) .}
\end{gathered}
$$

The previous theorem states that the STP-FE approximation has almost the same rate of convergence as the FTP-FE. On the other hand, the great advantage of the sparse approximation with respect to the full one is represented by a drastic reduction of the dimensionality of the sparse finite element space.

\section{Conclusions}

The present work addresses the mixed formulation of the Hodge Laplacian defined on an $n$-dimensional domain $D \subseteq \mathbb{R}^{n}(n \geqslant 1)$, with stochastic forcing terms. The well-posedness of this problem is equivalent to the inf-sup condition of a suitable bounded bilinear and symmetric form $\langle T \cdot, \cdot\rangle$ coming from the weak formulation of the mixed Hodge Laplacian.

We have studied the moment equations, that is, the deterministic equations solved by the statistical moments of the unique stochastic solution. In particular, if $T$ is the (deterministic) operator that defines the starting problem, we show that the $m$ th moment equation involves the tensor product operator $T^{\otimes m}:=\underbrace{T \otimes \cdots \otimes T}_{m \text { times }}$. The main achievement of the paper has been to characterize an operator $P$ and its tensorial version $P^{\otimes m}$ that allows us to construct suitable test functions to prove the inf-sup condition for the tensor problem $\left\langle T^{\otimes m} \cdot, \cdot\right\rangle$ both at the continuous level and at the discrete level with full or sparse FE discretizations. By this tool we have been able to show that known stable FE approximations for the deterministic problem are also stable and optimally convergent for the tensorial problem both in the full and sparse versions. 


\section{Funding}

The first and the third authors have been supported by the Italian grant FIRB-IDEAS (Project no. RBID08223Z) 'Advanced numerical techniques for uncertainty quantification in engineering and life science problems'.

\section{REFERENCES}

ARnold, D. N., FAlK, R. S. \& Winther, R. (2006) Finite element exterior calculus, homological techniques, and applications. Acta Numer, 15, 1-155.

ARNold, D. N., FALK, R. S. \& WinTHER, R. (2010) Finite element exterior calculus: from Hodge theory to numerical stability. Bull. Amer. Math. Soc. (N.S.), 47, 281-354.

BABUŠKA, I. \& AzIZ, A. K. (1972) Survey lectures on the mathematical foundations of the finite element method. The Mathematical Foundations of the Finite Element Method with Applications to Partial Differential Equations (Proc. Sympos., University of Maryland, Baltimore, MD, 1972). New York: Academic Press, pp. 1-359.

BABUŠKa, I. \& CHATZIPANTELIDIs, P. (2002) On solving elliptic stochastic partial differential equations. Comput. Methods Appl. Mech. Engrg., 191, 4093-4122.

BabušKa, I., Nobile, F. \& Tempone, R. (2007) A stochastic collocation method for elliptic partial differential equations with random input data. SIAM J. Numer. Anal., 45, 1005-1034.

Bäck, J., Nobile, F., TAMellini, L. \& Tempone, R. (2011) Stochastic spectral Galerkin and collocation methods for PDEs with random coefficients: a numerical comparison. Spectral and High Order Methods for Partial Differential Equations. Lecture Notes in Computational Science and Engineering, vol. 76. Berlin Heidelberg: Springer, pp. 43-62.

Barth, A., Schwab, C. \& Zollinger, N. (2011) Multi-level Monte Carlo finite element method for elliptic PDEs with stochastic coefficients. Numer. Math., 119, 123-161.

Bonizzoni, F. (2013) Analysis and approximation of moment equations for PDEs with stochastic data. Ph.D. thesis, Department of Mathematics, Politecnico di Milano, Italy.

Bonizzoni, F. \& Nobile, F. (2013) Perturbation analysis for the Darcy problem with log-normal permeability. Technical Report 38/2013. MOX - Modeling and Scientific Computing, Dipartimento di Matematica " $F$. Brioschi”, Politecnico di Milano.

Bossavit, A. (1998) Computational Electromagnetism. Variational Formulations, Complementarity, Edge Elements. San Diego, CA: Academic Press, pp. $\mathrm{xx}+352$.

Brezzi, F. \& Fortin, M. (1991) Mixed and Hybrid Finite Element Methods. Springer Series in Computational Mathematics, vol. 15. New York: Springer, pp. $x+350$.

BufFa, A. (2005) Remarks on the discretization of some noncoercive operator with applications to heterogeneous Maxwell equations. SIAM J. Numer. Anal., 43, 1-18 (electronic).

Bungartz, H.-J. \& Griebel, M. (2004) Sparse grids. Acta Numer., 13, 147-269.

Caflisch, R. E. (1998) Monte Carlo and quasi-Monte Carlo methods. Acta Numer., 7, 1-49.

Christiansen, S. H., Munthe-KaAs, H. Z. \& Owren, B. (2011) Topics in structure-preserving discretization. Acta Numer., 20, 1-119.

Christiansen, S. H. \& Winther, R. (2008) Smoothed projections in finite element exterior calculus. Math. Comp., 77, 813-829.

Cliffe, K. A., Giles, M. B., Scheichl, R. \& Tecrentrup, A. L. (2011) Multilevel Monte Carlo methods and applications to elliptic PDEs with random coefficients. Comput. Vis. Sci., 14, 3-15.

Cohen, A., Devore, R. \& Schwab, C. (2011) Analytic regularity and polynomial approximation of parametric and stochastic elliptic PDE's. Anal. Appl. (Singap.), 9, 11-47.

Delvos, F.-J. (1982) $d$-variate Boolean interpolation. J. Approx. Theory, 34, 99-114.

Fernandes, P. \& Gilardi, G. (1997) Magnetostatic and electrostatic problems in inhomogeneous anisotropic media with irregular boundary and mixed boundary conditions. Math. Models Methods Appl. Sci., 7, 957-991.

Franssen, H. J. H., Alcolea, A., Riva, M., Bakr, M., van der Wiel, M., Stauffer, F. \& Guadagnini, A. (2009) A comparison of seven methods for the inverse modelling of groundwater flow. Application to the characterisation of well catchments. Adv. Water Resour, 32, 851-872. 
GiLes, M. (2008a) Improved multilevel Monte Carlo convergence using the Milstein scheme. Monte Carlo and Quasi-Monte Carlo Methods 2006. Berlin: Springer, pp. 343-358.

Giles, M. B. (2008b) Multilevel Monte Carlo path simulation. Oper. Res., 56, 607-617.

Graham, I. G., Kuo, F. Y., Nuyens, D., Scheichl, R. \& Sloan, I. H. (2011) Quasi-Monte Carlo methods for elliptic PDEs with random coefficients and applications. J. Comput. Phys., 230, 3668-3694.

Guadagnini, A. \& Neuman, S. P. (1999a) Nonlocal and localized analyses of conditional mean steady state flow in bounded, randomly nonuniform domains: 1 . Theory and computational approach. Water Resour. Res., 35, 2999-3018.

Guadagnini, A. \& Neuman, S. P. (1999b) Nonlocal and localized analyses of conditional mean steady state flow in bounded, randomly nonuniform domains: 2. Computational examples. Water Resour. Res., 35, 3019-3039.

Harbrecht, H., Schneider, R. \& Schwab, C. (2008a) Multilevel frames for sparse tensor product spaces. Numer. Math., 110, 199-220.

Harbrecht, H., Schneider, R. \& Schwab, C. (2008b) Sparse second moment analysis for elliptic problems in stochastic domains. Numer. Math., 109, 385-414.

Heinrich, S. (2001) Multilevel Monte Carlo methods. Large-Scale Scientific Computing. Lecture Notes in Computer Science, vol. 2179. Berlin/Heidelberg: Springer, pp. 58-67.

Hiptmair, R. (2002) Finite elements in computational electromagnetism. Acta Numer., 11, 237-339.

Hiptmair, R., Schwab, C. \& Jerez-Hanckes, C. (2012) Sparse tensor edge elements. Technical Report 2012-34. Switzerland: Seminar for Applied Mathematics, ETH Zürich.

Kuo, F. Y., Schwab, C. \& Sloan, I. H. (2012) Quasi-Monte Carlo finite element methods for a class of elliptic partial differential equations with random coefficients. SIAM J. Numer. Anal., 50, 3351-3374.

Massey, W. S. (1991) A Basic Course in Algebraic Topology. Graduate Texts in Mathematics, vol. 127. New York: Springer, pp. xvi+428.

Monk, P. (2003) Finite Element Methods for Maxwell's Equations. Numerical Mathematics and Scientific Computation. New York: Oxford University Press, pp. xiv+450.

Niederreiter, H. (1992) Random Number Generation and Quasi-Monte Carlo Methods. CBMS-NSF Regional Conference Series in Applied Mathematics, vol. 63. Philadelphia, PA: Society for Industrial and Applied Mathematics (SIAM), pp. vi+241.

NovaK, E. \& RitTer, K. (1996) High dimensional integration of smooth functions over cubes. Numer. Math., 75, 79-97.

Reed, M. \& Simon, B. (1980) Methods of Modern Mathematical Physics: Functional Analysis, vol. I, 2nd edn. New York: Academic Press, pp. xv+400.

Riva, M., Guadagnini, A. \& De Simoni, M. (2006) Assessment of uncertainty associated with the estimation of well catchments by moment equations. Adv. Water Resour., 29, 676-691.

SCHÖBERL, J. (2008) A posteriori error estimates for Maxwell equations. Math. Comp., 77, 633-649.

Schwab, C. \& GitTelson, C. J. (2011) Sparse tensor discretizations of high-dimensional parametric and stochastic PDEs. Acta Numer., 20, 291-467.

Schwab, C. \& Todor, R. A. (2003) Sparse finite elements for elliptic problems with stochastic loading. Numer. Math., 95, 707-734.

Tartakovsky, D. M. \& Neuman, S. P. (1998) Transient flow in bounded randomly heterogeneous domains: 1. Exact conditional moment equations and recursive approximations. Water Resour. Res., 34, 1-12.

Teckentrup, A., Scheichl, R., Giles, M. \& Ullmann, E. (2013) Further analysis of multilevel Monte Carlo methods for elliptic PDEs with random coefficients. Numer. Math., 125, 569-600.

von Petersdorff, T. \& Schwab, C. (2006) Sparse finite element methods for operator equations with stochastic data. Appl. Math., 51, 145-180.

Zhang, D. (2002) Stochastic Methods for Flow in Porous Media Coping with Uncertainties. New York: Academic Press. 